\title{
LEVEL SET BASED MULTISPECTRAL SEGMENTATION WITH CORNERS *
}

\author{
WENHUA GAO ${ }^{\dagger}$ AND ANDREA BERTOZZI $\ddagger$
}

\begin{abstract}
In this paper we propose an active contour model for segmentation based on the Chan-Vese model. The new model can capture inherent sharp features, i.e., the sharp corners of objects, which are often smoothed by the regularization term in segmentation. Motivated by the snaked-based method in (Droske and Bertozzi SIAM J. on Image Sci. 2010) that emphasizes straight edges and corners without regard to orientation, we develop a region-based method with a level set representation. The model combines the Chan-Vese model with the level set version of a higher order nonlinear term. We extend this model to multispectral images. Higher order method can be very stiff, so we propose a splitting scheme to remove the stiffness and prove its stability and convergence. Finally we show numerical results on gray, color and hyperspectral images. We can see the model is robust to noise.
\end{abstract}

Key words. segmentation, corners, high order and nonlinear, level set representation, numerical stability and convergence

AMS subject classifications. 35G20, 65M06, 65M12

1. Introduction. Segmentation is one of the most important task in image processing. The main idea of image segmentation is to detect the objects in the given image. Usually, this is done by evolving a curve towards the boundary of the object. Generally speaking, the existing segmentation methods can be divided into two categories: curve based methods and region based methods. The curve based methods include the 'snake' model by Kass et al [21] and geodesic active contour model by Caselles et al [9]. The region based methods include Mumford-Shah [23] and related Chan-Vese [12] methods. We briefly describe these methods.

Kass et al (1988) [21] originated the 'snake' or active contour model. In the snake model, the curve evolution is obtained by minimizing a carefully designed functional energy. Let $\Omega$ be a bounded and open subset of $\mathbb{R}^{2}$, with $\partial \Omega$ its boundary. Let $f$ be the given image, as a bounded function defined on $\bar{\Omega}$ with real values. Usually $\bar{\Omega}$ is a rectangular domain. Let $C(q):[0,1] \rightarrow \mathbb{R}^{2}$ be a parametrized curve. Then the snake method is minimizing the following functional energy:

$$
E(C)=\alpha \int_{0}^{1}\left|C^{\prime}(q)\right|^{2} d q+\beta \int_{0}^{1}\left|C^{\prime \prime}(q)\right|^{2} d q-\lambda \int_{0}^{1}|\nabla f(C(q))|^{2} d q .
$$

The first two terms, the membrane energy and the elasticity energy, control the smoothness of the curve. They are called the internal energy. The third term is the external term and depends on the image data. It is easy to see that the external energy term is small when the gradient of $f$ has a large magnitude, thus pushing the curve towards edges. Such functions are usually called edge detectors. The active contour model was further developed by $[9,6,7,22,27]$ using different edge detectors. For example, Caselles et al [9] introduced a geodesic active contour model by

* This research is supported by ONR grant N000140810363, the Department of Defense and NSF grant DMS-0914856.

${ }^{\dagger}$ Department of Mathematics, University of California, Los Angeles, Los Angeles, CA 90095-1555 (wenhuagao@math.ucla.edu).

${ }^{\ddagger}$ Department of Mathematics, University of California, Los Angeles, Los Angeles, CA 90095-1555 (bertozzi@math.ucla.edu). 
minimizing the functional energy

$$
E_{g}(C)=\alpha \int_{0}^{1}\left|C^{\prime}(q)\right|^{2} d q+\lambda \int_{g}(|\nabla f(C(q))|)^{2} d q
$$

where $g: \mathbb{R}^{+} \rightarrow \mathbb{R}^{+}$is a decreasing function such that $\lim _{x \rightarrow \infty} g(x)=0$. In the geodesic active contour model, $-|\nabla f|^{2}$ is replaced by $g(f)^{2}$. In addition, considering that the snake model does not allow topology change in the curve evolution, and consequently can only detect one object in the image, Caselles et al employed a level set representation building on the pioneering work of Osher and Sethian [24]. Let $\phi(t, \cdot)$ be a level set function such that $C(q)$ is the zero level set of $\phi$, i.e., $C(q, t)=$ $\left\{x \in \mathbb{R}^{2}: \phi(t, x)=0\right\}$, then the level set function would be evolved instead of the curve. Caselles proposed the following equation for $\phi$

$$
\phi_{t}=|\nabla \phi| \operatorname{div}\left(g(f) \frac{\nabla \phi}{|\nabla \phi|}\right)+c g(f)|\nabla \phi|=g(c+\kappa)|\nabla \phi|+\nabla \phi \cdot \nabla g,
$$

where $\kappa$ is the curvature and $c$ is a constant.

Mumford and Shah [23] addressed an active contour model by minimizing the following energy

$$
E_{M S}(u, C)=\mu \operatorname{length}(C)+\lambda \int_{\Omega \backslash C}|u-f|^{2} d x+\int_{\Omega}|\nabla u|^{2} d x,
$$

where the boundary curve $C$ is exactly the discontinuity set of $u$. Chan and Vese [12] formulated a piecewise constant variant of this model, and the boundary curve $C$ was represented by a level set function $\phi$ satisfying $\phi>0$ inside $C$ and $\phi<0$ outside $C$. By defining the Heaviside function $H(x)=1_{x \geqslant 0}$ and the one-dimensional Dirac measure $\delta=\frac{d}{d x} H(x)$, the functional energy became

$$
\begin{aligned}
& E_{C V}\left(\phi, c_{1}, c_{2}\right)=\mu \int_{\Omega} \delta(\phi)|\nabla \phi|+\nu \int_{\Omega} H(\phi) d x d y \\
& \quad+\lambda_{1} \int_{\Omega}\left(f-c_{1}\right)^{2} H(\phi) d x d y+\lambda_{2} \int_{\Omega}\left(f-c_{2}\right)^{2}(1-H(\phi)) d x d y .
\end{aligned}
$$

The gradient descent equation for Chan and Vese active contour model is

$$
\begin{gathered}
c_{1}=\frac{\int_{\Omega} f H(\phi) d x d y}{\int_{\Omega} H(\phi) d x d y}, \quad c_{2}=\frac{\int_{\Omega} f(1-H(\phi)) d x d y}{\int_{\Omega}(1-H(\phi)) d x d y}, \\
\phi_{t}=\delta(\phi)\left[\mu \nabla \cdot \frac{\nabla \phi}{|\nabla \phi|}-\nu-\lambda_{1}\left(f-c_{1}\right)^{2}+\lambda_{2}\left(f-c_{2}\right)^{2}\right] .
\end{gathered}
$$

with boundary condition $\frac{\delta(\phi)}{\nabla \phi} \frac{\partial \phi}{\partial \vec{n}}=0$. This model was further extended in [11, 8]. Moreover, there are fast algorithms for solving the Chan-Vese model, including the method by Chambolle [10] and the split Bregman method by Goldstein and Osher [20].

All the segmentation models above minimize a functional energy including an edge detecting term and a regularization term which is usually the length term of the curve. As is known, the regularization terms can avoid local minima and ensure the 
smoothness of the boundary curve, especially when noise exists in the image. However, they often introduce undesired over-smoothing to the sharp features, especially corners. If the complete information about the morphology and anisotropy in the image is known, for example, the orientation of buildings in an aerial photograph, then it is natural to minimize some anisotropic functional to obtain segmentation with corners. This idea comes from the Wulff-shapes in material science. Numerical methods have been developed for anisotropic flows in $[1,5,14,15,16,18,19]$. However, the detection of Wulff-shape is a difficult problem. Consequently we focus on the automatic detection guided by the geometric features in the image.

Droske and Bertozzi [17] proposed a new algorithm based on the snake method and motivated by the low curvature image simplifier (LCIS), which is known for preserving jump discontinuity in slope. By combining the geodesic snake construction with nonlinear diffusion of edges, they are successful in segmenting objects with sharp corners. However, the method still suffers from other common drawbacks of snakebased methods. In particular one can not naturally perform topology changes and moreover, a multi scale preprocess of the image is required to avoid local minima due to cluster in the image. This prompt us to develop another segmentation model.

This paper is organized as follows. In the next section we first review the work of Droske and Bertozzi [17] with some discussion about the properties of the high order equations. Then we will formulate the corner preserving term in a level set representation. By combining the new corner preserving term with the Chan-Vese model, we obtain a new model that inherit the merits of Chan-Vese model as well as one that retains the sharp corners. In addition, we extend this model to the color and hyperspectral images. In section 3 we describe the numerical implementation details of the high order nonlinear PDE. We also prove the convergence of the time stepping scheme. In section 4 we validate our model by numerical test on gray, color and hyperspectral images, and we end the paper by a brief conclusion section.

2. Chan-Vese with corner preserving term. The new method developed in [17] is motivated the low - curvature image simplifier (LCIS), which is first introduced by Tumblin and Turk [31] and later developed by Bertozzi and Greer [3]. The key point is that the fourth-order scalar PDE

$$
u_{t}+\operatorname{div}(g(\Delta u) \nabla \Delta u)=0 .
$$

produces piecewise linear solutions and forms corners while smoothing out the noise. Here $g$ is typically a weight function, with $g(0)=1$ and $g(s) \rightarrow \infty$ as $s \rightarrow \infty$. In [31], the function $g$ was choosen as $g(s)=\left(1+\frac{s^{2}}{\eta^{2}}\right)^{-1}$ by analogy with the Perona-Malik method in [25], where $\eta$ is a positive parameter. The solution $u$ is actually a smooth function, thus the corner is understood in an infinitesimal sense. This equation is a gradient flow of the non-quadratic energy functional $E_{G}(u)=\int_{\Omega} G(\Delta u) d x$, where $G$ is the antiderivative of $g$. It also decreases the $H^{1}$ energy $E(u)=\int_{\Omega}|\nabla u|^{2} d x$. Bertozzi and Greer [3] proved the existence of global smooth solutions in 1 dimension case with the same choice of $g$. The equation (2.1) can be combined with an $L^{2}$ fidelity term obtaining a good denoiser of piecewise linear signals.

Droske and Bertozzi [17] introduces a straightforward geometric variant of equation (2.1) simply by replacing the differential operators by their corresponding intrinsically geometric counterparts and by choosing the coordinates $x$ as the free variable. They obtain the following PDE:

$$
x_{t}-\operatorname{div}_{\Gamma}\left(g(h) \nabla_{\Gamma} \Delta_{\Gamma} x\right)=0 .
$$


where $\nabla_{\Gamma}$, $\operatorname{div}_{\Gamma}, \Delta_{\Gamma}$ are the intrinsic surface gradient, surface divergence and surface Laplace operator respectively. Let $\vec{n}$ be the outer normal vector of the surface $\Gamma$ and $h$ be the mean curvature. Note that $\Delta_{\Gamma} x=h \vec{n}$, the equation (2.2) is quite similar to the equation that describes the evolution of surface under surface diffusion with a mobility function $g(s)$ that depends on the scalar mean curvature $h$ :

$$
x_{t}-\operatorname{div}_{\Gamma}\left(g(h) \nabla_{\Gamma} h\right) \vec{n}=0 .
$$

Like the regular surface diffusion, equation (2.3) can preserve the area enclosed by $\Gamma$ and decrease the length of $\Gamma$. The derivation for the area preservation and length decreasing is similar to that of a regular domain.

$$
\begin{gathered}
\frac{d}{d t}|A(t)|=\int_{\Gamma(t)} v d s=\int_{\Gamma(t)} \operatorname{div}\left(g(h) \nabla_{\Gamma} h\right) d s=-\int_{\Gamma(t)} g(h) \nabla_{\Gamma} h \cdot \nabla_{\Gamma} 1 d s=0 . \\
\frac{d}{d t}|\Gamma(t)|=\int_{\Gamma(t)} v h d s=\int_{\Gamma(t)} \operatorname{div}\left(g(h) \nabla_{\Gamma} h\right) h d s=-\int_{\Gamma(t)} g(h) \nabla_{\Gamma} h \cdot \nabla_{\Gamma} h d s \leqslant 0 .
\end{gathered}
$$

Therefore, we can use (2.3) to replace the length regularization term in active contour models, although [17] uses (2.2) for simpler numerical implementation.

The main purpose of this manuscript is to recast this equation in terms of a level set formulation, and to illustrate its usefulness in segmenting complex images with sharp corners. Following the level set representation of the geometric features in Chopp et al [13] and Bertamio et al [2], we obtain the level set version, which is fourth order and nonlinear. While the derivation of the equation is straightforward, the main challenge in numerical implementation is to develop an efficient time stepping scheme. For example, explicit schemes usually requires that $d t \sim d x^{4}$, which is very stiff. In section 3 we propose an efficient splitting scheme and prove its convergence.

Suppose the initial surface is given by the zero level set of a function $\phi(\cdot, 0)$, or, $\Gamma(0)=\{x: \phi(x, 0)=0\}$, and the surface at time $t$ is the zeros level set of $\phi(\cdot, t)$. The normal direction is given by $\vec{n}=\frac{\nabla \phi}{|\nabla \phi|}$ and the mean curvature $h=\operatorname{div}(\vec{n})$ for any point on the curve $\Gamma$. Further we need to define the intrinsic surface gradient, surface divergence and surface Laplacian operator via level set representation. In [2], Bertalmio et al derived all these operators via level set representation and solved PDE on surfaces. According to their work, the surface gradient is simply the projection of the gradient operator onto the tangent plane:

$$
\nabla_{\Gamma} \phi=\nabla \phi-(\nabla \phi \cdot \vec{n}) \vec{n}
$$

The surface gradient operator $\operatorname{div}_{\Gamma}$ is the dual operator of the surface gradient operator, and the surface Laplacian, or the Laplace-Beltrami operator is given by

$$
\Delta_{\Gamma} \phi=\operatorname{div}_{\Gamma}\left(\nabla_{\Gamma} \phi\right)
$$

Now we only need to convert the corner preserving equation into a level set formulation. We are more interested in equation (2.3) than (2.2), because of the length decreasing property and simpler numerical implementation. With all the level representation above, the level set version of equation (2.3) can be written as:

$$
\phi_{t}=-|\nabla \phi| \operatorname{div}_{\Gamma}\left(g(h) \nabla_{\Gamma} h\right)
$$


To demonstrate the curve evolution by equation (2.6), we repeat the following numerical curve evolution test in Droske et al [17]. The initial curve is chosen in polar coordinate as $r=\frac{1}{2}+\frac{1}{10} \sin (15 \theta)$, where $r$ and $\theta$ are just the classic polar coordinate parameters: $r=\sqrt{x^{2}+y^{2}}, \theta=\arctan \frac{y}{x}$. The initial level set function is $u=r-\frac{1}{2}-\frac{1}{10} \sin (15 \theta)$. The curve evolution is shown in Figure (2.1). Using level set representation, the black curve is the zeros level set of the function $\phi$ and the color in the figure stands for the value of the level set function. During the evolution, the initial smooth curve develops corners quickly by accentuating the high curvature parts. The corners keep existing until the curve converges to a circle by the infinitesimal regularity.
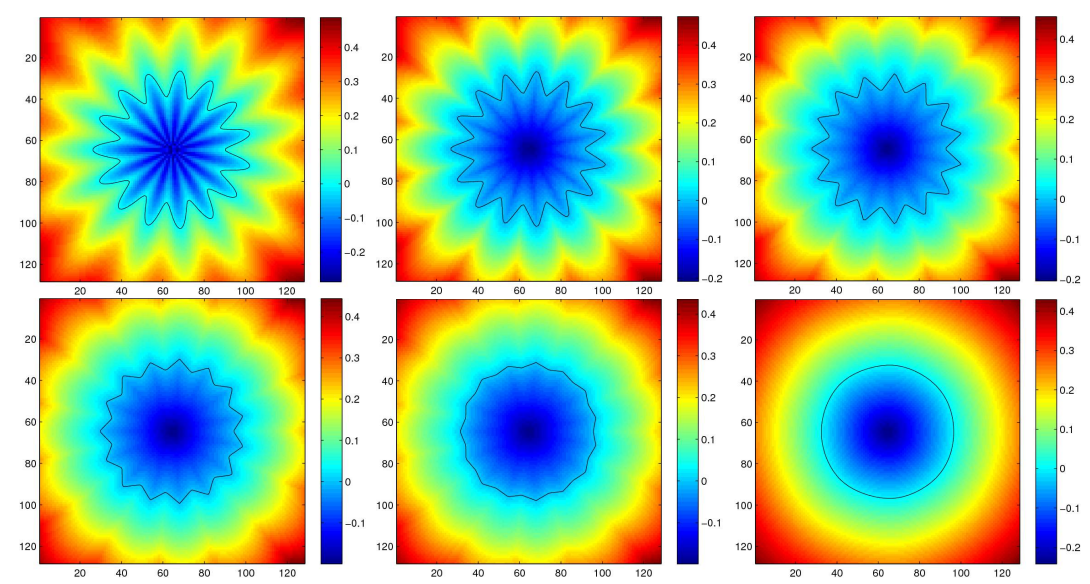

FIG. 2.1. The evolution of a curve. We can see that corners are formed in early stage.

The idea of this manuscript comes from the process above. If the curve evolution is combined with a fidelity term, we can expect the curve to stop at a stable state with sharp corners. This prompts us to combine the Chan-Vese model with the equation (2.6). With the fitting term in Chan-Vese model, we get the following equation.

$$
\begin{aligned}
\phi_{t} & =-\alpha|\nabla \phi| \operatorname{div}_{\Gamma}\left(g(h) \nabla_{\Gamma} h\right) \\
& +\delta(\phi)\left[\mu \nabla \cdot \frac{\nabla \phi}{|\nabla \phi|}-\nu-\lambda_{1}\left(f-c_{1}\right)^{2}+\lambda_{2}\left(f-c_{2}\right)^{2}\right] .
\end{aligned}
$$

For multiband images, let $N$ be the number of bands and $f_{i}$ be the gray value of the $i$ th band. Using the technique in $[27,11]$, we can similarly calculate the $c_{1 i}$ and $c_{2 i}$ of the $i$ th band with $f_{i}$ and the level set function $\phi$, and then we obtain the level set evolution equation for multi-band images by simply taking the algebraic average of the gradient descent flow for each band. This can also be combined with segmentation method with spectral angle by Ye [32], in which the authors used spectral angle for hyperspectral images instead of the Chan-Vese fidelity term.

$$
\begin{aligned}
\phi_{t} & =-\alpha|\nabla \phi| \operatorname{div}_{\Gamma}\left(g(h) \nabla_{\Gamma} h\right) \\
& +\delta(\phi)\left[\mu \nabla \cdot \frac{\nabla \phi}{|\nabla \phi|}-\nu-\frac{1}{N} \sum_{i=1}^{N} \lambda_{1 i}\left(f_{i}-c_{1 i}\right)^{2}+\frac{1}{N} \sum_{i=1}^{N} \lambda_{2 i}\left(f_{i}-c_{2 i}\right)^{2}\right] .
\end{aligned}
$$

As we mentioned above, the corner preserving term can decrease the curve length and impose regularization on the level set function. Therefore, we can drop the length 
term in Chan-Vese model and only use the corner preserving term. Solving high order nonlinear is usually difficult, since the stability condition is more restrictive. We will describe the numerical scheme in the next section.

3. Semi-Implicit Numerical Scheme. Equation (2.7) and (2.6) are fourth order nonlinear equations. For the numerical implementation, if we apply an explicit numerical scheme, the nonlinear high order equation usually requires a time step $d t \sim$ $d x^{4}$, which leads to very slow convergence. If we attempt a fully implicit numerical scheme, then solving the nonlinear implicit equation at each time step is difficult. As a result, semi-implicit schemes are preferred for this kind of equations. We consider the numerical scheme introduced by Smereka, Salac and Lu [26, 29] for the curve evolution by surface Laplacian of mean curvature. Although this method has been discovered and implemented numerically in the literature in these papers, a rigorous proof of convergence remains new. We extend the scheme in [26, 29] to general cases, and prove convergence of the time stepping scheme.

For simplicity, we write the equation (2.7) as $\phi_{t}=S(\phi)$. We add a bilaplacian stabilization term to both sides of the PDE and obtain

$$
\phi_{t}+\beta \Delta^{2} \phi=S(\phi)+\beta \Delta^{2} \phi
$$

with $\beta$ a positive constant. To distinguish the exact solution from the numerical solution, we use upper case and bold characters for the numerical solution, lower case for the exact solution. In other words, we write $\Phi^{k}, \mathbf{h}^{k}, \nabla_{\boldsymbol{\Gamma}}$ and $\Delta_{\boldsymbol{\Gamma}}$ for the numerical equation at the $k$ th step, while $\phi^{k}, h^{k}, \nabla_{\Gamma}$ and $\Delta_{\Gamma}$ for the exact solution at time $k \cdot d t$. Let $e^{k}=\phi^{k}-\Phi^{k}$ denote the discretization error. Taking the left side bilaplacian at the new time level and the entire right side at the old time level, we obtain

$$
\frac{\Phi^{k+1}-\Phi^{k}}{d t}+d t \cdot \beta \Delta^{2} \Phi^{k+1}=d t\left(\beta \Delta^{2} \Phi^{k}+S\left(\Phi^{k}\right)\right),
$$

which is equivalent to

$$
\left(\Phi^{k+1}-\Phi^{k}\right)=d t\left(1+d t \cdot \beta \Delta^{2}\right)^{-1} S\left(\Phi^{k}\right) .
$$

For image processing problems, we usually take the domain $\Omega=[0,1) \times[0,1)$. In the following part we outline the discretization of equation (2.7). The right hand side is composed of two parts, the Chan-Vese energy term and the corner preserving term. For the Chan-Vese energy term, we simply follow the numerical discretization in [12]. We focus on the corner preserving term.

First, the outer normal direction $\overrightarrow{\mathbf{n}}$ is

$$
\overrightarrow{\mathbf{n}}=\left(\mathbf{n}^{x}, \mathbf{n}^{y}\right)=\frac{\nabla \Phi}{|\nabla \Phi|}=\left(\frac{\Phi_{x}}{\left(\Phi_{x}^{2}+\Phi_{y}^{2}\right)^{1 / 2}}, \frac{\Phi_{y}}{\left(\Phi_{x}^{2}+\Phi_{y}^{2}\right)^{1 / 2}}\right)
$$

Then the mean curvature can be represented as

$$
\begin{aligned}
\mathbf{h} & =\operatorname{div}(\overrightarrow{\mathbf{n}})=\frac{\Delta \Phi}{|\nabla \Phi|}-\frac{\nabla \Phi^{T} \nabla^{2} \Phi \nabla \Phi}{|\nabla \Phi|^{3}} \\
& =\frac{\Phi_{x x}+\Phi_{y y}}{\left(\Phi_{x}^{2}+\Phi_{y}^{2}\right)^{1 / 2}}-\frac{\Phi_{x}^{2} \Phi_{x x}+2 \Phi_{x} \Phi_{y} \Phi_{x y}+\Phi_{y}^{2} \Phi_{y y}}{\left(\Phi_{x}^{2}+\Phi_{y}^{2}\right)^{3 / 2}} .
\end{aligned}
$$


In the actual implementation, we usually use $|\nabla \Phi|_{\delta}=\left(\Phi_{x}^{2}+\Phi_{y}^{2}+\delta^{2}\right)^{1 / 2}$ instead of $|\nabla \Phi|=\left(\Phi_{x}^{2}+\Phi_{y}^{2}\right)^{1 / 2}$ to avoid division by zero, where $\delta$ is a small parameter. Consequently the modified normal direction and mean curvature are

$$
\begin{aligned}
\overrightarrow{\mathbf{n}}_{\delta}=\left(\mathbf{n}_{\delta}^{x}, \mathbf{n}_{\delta}^{y}\right) & =\frac{\nabla \Phi}{|\nabla \Phi|_{\delta}}=\left(\frac{\Phi_{x}}{\left(\Phi_{x}^{2}+\Phi_{y}^{2}+\delta^{2}\right)^{1 / 2}}, \frac{\Phi_{y}}{\left(\Phi_{x}^{2}+\Phi_{y}^{2}+\delta^{2}\right)^{1 / 2}}\right) . \\
\mathbf{h}_{\delta} & =\operatorname{div}\left(\overrightarrow{\mathbf{n}}_{\delta}\right)=\frac{\Delta \Phi}{|\nabla \Phi|_{\delta}}-\frac{\nabla \Phi^{T} \nabla^{2} \Phi \nabla \Phi}{|\nabla \Phi|_{\delta}^{3}} \\
& =\frac{\Phi_{x x}+\Phi_{y y}}{\left(\Phi_{x}^{2}+\Phi_{y}^{2}+\delta^{2}\right)^{1 / 2}}-\frac{\Phi_{x}^{2} \Phi_{x x}+2 \Phi_{x} \Phi_{y} \Phi_{x y}+\Phi_{y}^{2} \Phi_{y y}}{\left(\Phi_{x}^{2}+\Phi_{y}^{2}+\delta^{2}\right)^{3 / 2}} .
\end{aligned}
$$

For numerical analysis, we make the same modification for the original equations (2.6) and (2.7), i.e., we use the modified $|\nabla \phi|_{\delta}=\left(\phi_{x}^{2}+\phi_{y}^{2}+\delta^{2}\right)^{1 / 2}$ instead of $|\nabla \phi|=$ $\left(\phi_{x}^{2}+\phi_{y}^{2}\right)^{1 / 2}$, and consequently use modified $\vec{n}_{\delta}$ and $h_{\delta}$ instead of $\vec{n}$ and $h$. As long as the parameter $\delta$ is small enough, the zero level set of the modified equation is a good approximation to that of the original equation. In addition, the modified equation can avoid the singularity at the local maxima and minima of the level set function $\phi$. Therefore, from now on we always discuss the modified equations (2.6) and (2.7). Since the main difficulty for numerical implementation is the surface Laplacian term, which is fourth order and nonlinear, we focus on the equation (2.6) rather than equation (2.7). The modified equation and corresponding numerical scheme goes as follows.

$$
\begin{gathered}
\phi_{t}=-|\nabla \phi|_{\delta} \operatorname{div}_{\Gamma}\left(g\left(h_{\delta}\right) \nabla_{\Gamma} h_{\delta}\right) \\
\frac{\Phi^{k+1}-\Phi^{k}}{d t}+\beta \Delta^{2} \Phi^{k+1}=\beta \Delta^{2} \Phi^{k}-\left|\nabla \Phi^{k}\right|_{\delta} \operatorname{div}_{\Gamma}\left(g\left(\mathbf{h}_{\delta}^{k}\right) \nabla_{\boldsymbol{\Gamma}} \mathbf{h}_{\delta}^{k}\right) .
\end{gathered}
$$

To compute $\operatorname{div}_{\boldsymbol{\Gamma}}\left(g\left(\mathbf{h}_{\delta}\right) \nabla_{\boldsymbol{\Gamma}} \mathbf{h}_{\delta}\right)$, we may take the surface gradient of the mean curvature $\mathbf{h}_{\delta}$, and then calculate the surface divergence of $g\left(\mathbf{h}_{\delta}\right) \nabla_{\Gamma} \mathbf{h}_{\delta}$. However, we prefer to calculate the surface Laplacian of $G(\mathbf{h})$ as in [3], where $G$ is the antiderivative of $g$, or, $G^{\prime}(s)=g(s)$. In our numerical method, we choose $g(s)=\left(1+\frac{s^{2}}{\eta^{2}}\right)^{-1}$ and $G(s)=\frac{1}{\eta} \arctan \left(\frac{s}{\eta}\right)$ where $\eta$ is a positive parameter. According to the definition of surface gradient $\nabla_{\boldsymbol{\Gamma}} G=\nabla G-\left(\nabla G \cdot \overrightarrow{\mathbf{n}_{\delta}}\right) \overrightarrow{\mathbf{n}_{\delta}}$, we have the following component form $\left(\nabla G \cdot \overrightarrow{\mathbf{n}_{\delta}}\right)=\mathbf{n}_{\delta}^{x} G_{x}+\mathbf{n}_{\delta}^{y} G_{y}$, where the subscripts on $G$ denotes the partial derivatives in $x$ and $y$ individually. Therefore we can write the surface gradient as

$$
\begin{aligned}
\nabla_{\boldsymbol{\Gamma}} G & =G_{x} e^{x}+G_{y} e^{y}-\left(\mathbf{n}_{\delta}^{x} G_{x}+\mathbf{n}_{\delta}^{y} G_{y}\right)\left(\mathbf{n}_{\delta}^{x} e^{x}+\mathbf{n}_{\delta}^{y} e^{y}\right) \\
& \equiv A e^{x}+B e^{y}
\end{aligned}
$$

where $e^{x}$ and $e^{y}$ are unit vectors in the $x$ and $y$ direction respectively. By computing the surface divergence in a similar way we can obtain the surface Laplacian of $G\left(\mathbf{h}_{\delta}\right)$

$$
\Delta_{\Gamma} G=A_{x}+B_{y}-\mathbf{n}_{\delta}^{x}\left(\mathbf{n}_{\delta}^{x} A_{x}+\mathbf{n}_{\delta}^{y} A_{y}\right)-\mathbf{n}_{\delta}^{y}\left(\mathbf{n}_{\delta}^{x} B_{x}+\mathbf{n}_{\delta}^{y} B_{y}\right) .
$$

Next we will analyze this semi-implicit scheme with some more details and rigorous estimates for the numerical solution. We use similar technique as in Bertozzi et 
al [4] and Schoenlieb et al [28], focusing on discretization in time. Denote $\left|D^{m} u\right|^{2}=$ $\sum_{|\alpha|=m}\left|\partial^{\alpha} u\right|^{2}$ and $\left\|D^{m} u\right\|^{2}=\sum_{|\alpha|=m}\left\|\partial^{\alpha} u\right\|^{2}$ for any integer $m$, where $\alpha=\left(\alpha_{1}, \alpha_{2}\right)$, $|\alpha|=\alpha_{1}+\alpha_{2}, \partial^{\alpha}=\frac{\partial^{\alpha_{1}+\alpha_{2}}}{\partial x^{\alpha_{1}} \partial y^{\alpha_{2}}}$. Due to the high order and nonlinearity, we need several restrictions on the smoothness of the level set function. The results are summarized in the following theorem.

THEOREM 3.1. Let $\phi$ be the exact solution of (3.8) and $\phi^{k}=\phi(k d t)$ be the exact solution at time $k d t$ for a time step $d t>0$ and $k \in \mathbb{N}$. Let $\Phi^{k}$ be the kth iterate of (3.9). Assume that there exits a constant $L$ such that $|g(s)| \leqslant L,\left|g^{\prime}(s)\right| \leqslant L$, and the discrete solution exists up to time $T$, then we have the following statements:

(i) Under the assumption that $\left\|\phi_{t t}\right\|_{-1},\left\|\nabla \Delta \phi_{t}\right\|_{2},\|\nabla \phi\|_{\infty}$ and $\left\|\phi_{t}\right\|_{-1}$ are bounded, the numerical scheme (3.9) is consistent with the modified continuous equation (3.8) and first order in time.

(ii) Let further $e^{k}=\phi^{k}-\Phi^{k}$ be the discretization error. If

$$
\left\|\partial^{\alpha} \phi^{k}\right\|_{\infty} \leqslant K, \quad\left\|\nabla \Phi^{k}\right\|_{\infty} \leqslant K
$$

for a constant $K>0$ and all $|\alpha| \leqslant 3, k d t \leqslant T$, then the error $e^{k}$ converges to zero with first order in time.

REMARK: 1. Although the following convergence proof only requires $d t$ smaller than some constant which is independent of $d x$, the assumption that the derivatives of $\phi$ are bounded may impose additional restriction on the time step $d t$. In fact, for the most commonly used level set function, the signed distance function, $|\nabla \phi|$ is usually unbounded. In addition, all the constants depend on the choice of $\delta$. However, we have to take $\delta$ small to make sure that the solution of the modified equation is close to the solution of the original equation. We may have to take $d t$ small enough to obtain desired accuracy.

REMARK: 2. Solving the equation in a narrow band of the zero level set may reduce the singularity of the level set function. For example, the signed distance function is singular in the whole domain, but it is smooth in a small neighborhood of the zero level set, as long as the zero level curve is smooth. In addition, in the numerical implementation, we impose an upper bound $K$ for $|\nabla \Phi|$. As soon as $|\nabla \Phi|$ exceeds $K$, we reinitialize the level set function.

The proof of the theorem above is split into three propositions. We first introduce the following lemmas, and then state the three propositions.

LEMMA 3.2. Let $\phi$ be a smooth function and surface $\Gamma=\{(x, y): \phi(x, y)=0\}$ be the zero level set of $\phi$. Then for any function $u, v \in L_{2}(\Omega)$, the modified surface gradient operator $\nabla_{\Gamma}$ satisfies

$$
\left|\nabla_{\Gamma} u\right|^{2} \leqslant|\nabla u|^{2} \leqslant|\nabla u|_{\delta}^{2}
$$

Proof. If we use the original $\nabla_{\Gamma} \phi$, then it is the projection of $\nabla \phi$ on the tangent plane, the inequality means the length of the projection is smaller than the original vector, which is true automatically. But the modified operator is no longer projection. However, the modified operator satisfies

$$
\nabla_{\Gamma} u=\nabla u-\left(\nabla u \cdot \vec{n}_{\delta}\right) \vec{n}_{\delta},
$$


Therefore, we have

$$
\begin{aligned}
\left|\nabla_{\Gamma} u\right|^{2} & =\nabla_{\Gamma} u \cdot \nabla_{\Gamma} u \\
& =\left(\nabla u-\left(\nabla u \cdot \vec{n}_{\delta}\right) \vec{n}_{\delta}\right) \cdot\left(\nabla u-\left(\nabla u \cdot \vec{n}_{\delta}\right) \vec{n}_{\delta}\right) \\
& =\nabla u \cdot \nabla u-2\left(\nabla u \cdot \vec{n}_{\delta}\right)^{2}+\left(\nabla u \cdot \vec{n}_{\delta}\right)^{2}\left(\vec{n}_{\delta} \cdot \vec{n}_{\delta}\right) \\
& =|\nabla u|^{2}-\left(\nabla u \cdot \vec{n}_{\delta}\right)^{2}\left(2-\left|\vec{n}_{\delta}\right|^{2}\right) \\
& \leqslant|\nabla u|^{2} \leqslant|\nabla u|_{\delta}^{2} .
\end{aligned}
$$

by the fact $0 \leqslant\left|\vec{n}_{\delta}\right|^{2} \leqslant|\vec{n}|^{2}=1$. In addition, we can verify this is also true for the discretized solution.

LEMMA 3.3. We have the following inequalities

$$
\begin{aligned}
& \left\|D^{2} u\right\|_{2}^{2} \leqslant\|\Delta u\|_{2}^{2} \leqslant 2\left\|D^{2} u\right\|_{2}^{2}, \\
& \left\|D^{3} u\right\|_{2}^{2} \leqslant\|\nabla \Delta u\|_{2}^{2} \leqslant 3\left\|D^{3} u\right\|_{2}^{2}, \\
& \left\|D^{2} u\right\|_{2}^{2} \leqslant\|\nabla u\|_{2}+\left\|D^{3} u\right\|_{2} .
\end{aligned}
$$

Proof. Integrate by parts for $\|\Delta u\|_{2}^{2}$ and we obtain

$$
\|\Delta u\|_{2}^{2}=\int\left(u_{x x}^{2}+2 u_{x x} u_{y y}+u_{y y}^{2}\right) d x d y=\int\left(u_{x x}^{2}+2 u_{x y}^{2}+u_{y y}^{2}\right) d x d y .
$$

The second part can be verified in a similar way. For the third part, we have

$$
\int u_{x x}^{2} d x d y=-\int u_{x} u_{x x x} d x d y \leqslant \frac{1}{2}\left(\int u_{x}^{2} d x d y+\int u_{x x x}^{2} d x d y\right) .
$$

Do the same to $\int u_{x y}^{2} d x d y$ and $\int u_{y y}^{2} d x d y$, we can come to the conclusion.

LEMma 3.4. For any $u$, there exist some constant $C=C(\Omega)$ such that

$$
\left\|D^{2} u\right\|_{4}^{2} \leqslant C\|\nabla u\|_{\infty}\left\|D^{3} u\right\|_{2} .
$$

Proof. By Gagliardo-Nirenberg inequality as in [30], for any $f$ we have

$$
\|D f\|_{4}^{2} \leqslant C\|f\|_{\infty}\left\|D^{2} f\right\|_{2} .
$$

By taking $f=\Phi_{x}$ and $f=\Phi_{y}$ we obtain the inequality.

Proposition 3.5. (Consistency) Under the same assumptions as in Theorem 3.1, the numerical scheme (3.9) is consistent with equation (2.6) with local truncation error $\left\|\tau^{k}\right\|_{-1}=O(d t)$.

Proof. The local truncation error is defined as

$$
\tau^{k}=\frac{\phi^{k+1}-\phi^{k}}{d t}+\beta \Delta^{2}\left(\phi^{k+1}-\phi^{k}\right)-\left|\nabla \phi^{k}\right|_{\delta} \nabla_{\Gamma}\left(g\left(h_{\delta}^{k}\right) \nabla_{\Gamma} h_{\delta}^{k}\right) .
$$

Taking the Taylor series of $\phi$ at $k d t$ and assuming that $\left\|\phi_{t t}\right\|_{-1},\left\|\nabla \Delta \phi_{t}\right\|_{2},\|\nabla \phi\|_{\infty}$ and $\left\|\phi_{t}\right\|_{-1}$ are bounded, we obtain that

$$
\left\|\tau^{k}\right\|_{-1}=O(d t)
$$

thus the local truncation error is first order in time. 
Proposition 3.6. (Stability) Under the same assumptions as Theorem 3.1 and assume $\left\|\nabla \Phi^{k}\right\|_{\infty} \leqslant K$ for all $k d t \leqslant T$, then the numerical solution $\Phi^{k}$ satisfies

$$
\left\|\nabla \Phi^{k}\right\|_{2}^{2}+d t K_{1}\left\|\nabla \Delta \Phi^{k}\right\|_{2}^{2} \leqslant e^{K_{2} T}\left(\left\|\nabla \Phi_{0}\right\|_{2}^{2}+d t K_{1}\left\|\nabla \Delta \Phi_{0}\right\|_{2}^{2}\right),
$$

for some constant $K_{1}, K_{2}$.

Proof. We multiply (3.9) with $\Delta \Phi^{k+1}$ and integrate over $\Omega$, then we obtain

$$
\begin{aligned}
\frac{\left\langle\Phi^{k+1}, \Delta \Phi^{k+1}\right\rangle-\left\langle\Phi^{k}, \Delta \Phi^{k+1}\right\rangle}{d t} & +\beta\left(\left\langle\Delta^{2} \Phi^{k+1}, \Delta \Phi^{k}\right\rangle-\left\langle\Delta^{2} \Phi^{k+1}, \Delta \Phi^{k}\right\rangle\right) \\
& =-\left\langle\operatorname{div}_{\boldsymbol{\Gamma}}\left(g\left(\mathbf{h}_{\delta}^{k}\right) \nabla_{\boldsymbol{\Gamma}} \mathbf{h}_{\delta}^{k}\right),\left|\nabla \Phi^{k}\right|_{\delta} \Delta \Phi^{k+1}\right\rangle .
\end{aligned}
$$

Integrate by parts for both sides, then we obtain

$$
\begin{aligned}
\frac{\left\langle\nabla \Phi^{k+1}, \nabla \Phi^{k+1}\right\rangle-\left\langle\nabla \Phi^{k}, \nabla \Phi^{k+1}\right\rangle}{d t} & +\beta\left(\left\|\nabla \Delta \Phi^{k+1}\right\|_{2}^{2}-\left\langle\nabla \Delta \Phi^{k+1}, \nabla \Delta \Phi^{k}\right\rangle\right) \\
& =-\left\langle g\left(\mathbf{h}_{\delta}^{k}\right) \nabla_{\boldsymbol{\Gamma}} \mathbf{h}_{\delta}^{k}, \nabla_{\boldsymbol{\Gamma}}\left(\left|\nabla \Phi^{k}\right|_{\delta} \Delta \Phi^{k+1}\right)\right\rangle .
\end{aligned}
$$

Applying Cauchy's inequality we obtain

$$
\left\langle\Phi^{k+1}, \Phi^{k}\right\rangle \leqslant \frac{1}{2}\left(\left\|\Phi^{k+1}\right\|_{2}^{2}+\left\|\Phi^{k}\right\|_{2}^{2}\right) .
$$

Consequently we have

$$
\left\|\Phi^{k+1}\right\|_{2}^{2}-\left\langle\Phi^{k+1}, \Phi^{k}\right\rangle \leqslant \frac{1}{2}\left(\left\|\Phi^{k+1}\right\|_{2}^{2}-\left\|\Phi^{k}\right\|_{2}^{2}\right) .
$$

Similarly we have

$$
\left\|\Delta \Phi^{k+1}\right\|_{2}^{2}-\left\langle\Delta \Phi^{k+1}, \Delta \Phi^{k}\right\rangle \leqslant \frac{1}{2}\left(\left\|\Delta \Phi^{k+1}\right\|_{2}^{2}-\left\|\Delta \Phi^{k}\right\|_{2}^{2}\right) .
$$

Therefore, we obtain the following inequality by lemma 3.2.

$$
\begin{aligned}
\frac{\left\|\nabla \Phi^{k+1}\right\|_{2}^{2}-\left\|\nabla \Phi^{k}\right\|_{2}^{2}}{2 d t} & +\frac{\beta}{2}\left(\left\|\nabla \Delta \Phi^{k+1}\right\|_{2}^{2}-\left\|\nabla \Delta \Phi^{k}\right\|_{2}^{2}\right) \\
& =-\left\langle g\left(\mathbf{h}_{\delta}^{k}\right)\left|\nabla \Phi^{k}\right|_{\delta} \nabla_{\boldsymbol{\Gamma}} \mathbf{h}_{\delta}^{k}, \nabla_{\boldsymbol{\Gamma}}\left(\left|\nabla \Phi^{k}\right|_{\delta} \Delta \Phi^{k+1}\right) /\left|\nabla \Phi^{k}\right|_{\delta}\right\rangle \\
& \leqslant \frac{1}{2 \varepsilon}\left\|g\left(\mathbf{h}_{\delta}^{k}\right)\left|\nabla \Phi^{k}\right|_{\delta} \nabla_{\boldsymbol{\Gamma}} \mathbf{h}_{\delta}^{k}\right\|_{2}^{2}+\frac{\varepsilon}{2}\left\|\nabla_{\boldsymbol{\Gamma}}\left(\left|\nabla \Phi^{k}\right|_{\delta} \Delta \Phi^{k+1}\right) /\left|\nabla \Phi^{k}\right|_{\delta}\right\|_{2}^{2} \\
& \leqslant \frac{L}{2 \varepsilon}\left\|\left|\nabla \Phi^{k}\right|_{\delta} \nabla_{\boldsymbol{\Gamma}} \mathbf{h}_{\delta}^{k}\right\|_{2}^{2}+\frac{\varepsilon}{2}\left\|\nabla_{\boldsymbol{\Gamma}}\left(\left|\nabla \Phi^{k}\right|_{\delta} \Delta \Phi^{k+1}\right) /\left|\nabla \Phi^{k}\right|_{\delta}\right\|_{2}^{2} \\
& \leqslant \frac{L}{2 \varepsilon}\left\|\left|\nabla \Phi^{k}\right|_{\delta} \nabla \mathbf{h}_{\delta}^{k}\right\|_{2}^{2}+\frac{\varepsilon}{2}\left\|\nabla\left(\left|\nabla \Phi^{k}\right|_{\delta} \Delta \Phi^{k+1}\right) /\left|\nabla \Phi^{k}\right|_{\delta}\right\|_{2}^{2} .
\end{aligned}
$$

Then we estimate $\nabla \mathbf{h}_{\delta}^{k}$. Similar to the original level set representation of mean curvature $h$, the modified $h_{\delta}$ has the following representation.

$$
\mathbf{h}_{\delta}^{k}=\nabla \cdot\left(\frac{\nabla \Phi^{k}}{\left|\nabla \Phi^{k}\right|_{\delta}}\right)=\frac{\Delta \Phi^{k}}{\left|\nabla \Phi^{k}\right|_{\delta}}-\frac{\left(\nabla \Phi^{k}\right)^{T} \nabla^{2} \Phi^{k} \nabla \Phi^{k}}{\left|\nabla \Phi^{k}\right|_{\delta}^{3}} .
$$

and

$$
\begin{aligned}
\left|\nabla \Phi^{k}\right|_{\delta} \nabla \mathbf{h}_{\delta}^{k} & =\left|\nabla \Phi^{k}\right|_{\delta} \nabla\left(\frac{\Delta \Phi^{k}}{\left|\nabla \Phi^{k}\right|_{\delta}}-\frac{\left(\nabla \Phi^{k}\right)^{T} \nabla^{2} \Phi^{k} \nabla \Phi^{k}}{\left|\nabla \Phi^{k}\right|_{\delta}^{3}}\right) \\
& =\nabla \Delta \Phi^{k}-\frac{\Delta \Phi^{k} \nabla^{2} \Phi^{k} \nabla \Phi^{k}}{\left|\nabla \Phi^{k}\right|_{\delta}^{2}}-\frac{2 \nabla^{2} \Phi^{k} \nabla^{2} \Phi^{k} \nabla \Phi^{k}}{\left|\nabla \Phi^{k}\right|_{\delta}^{2}} \\
& -\frac{\nabla \Phi^{k} \nabla\left(\nabla^{2} \Phi^{k}\right) \nabla \Phi^{k}}{\left|\nabla \Phi^{k}\right|_{\delta}^{2}}+\frac{3\left(\nabla \Phi^{k}\right)^{T} \nabla^{2} \Phi^{k} \nabla \Phi^{k} \nabla^{2} \Phi^{k} \nabla \Phi^{k}}{\left|\nabla \Phi^{k}\right|_{\delta}^{4}} .
\end{aligned}
$$


In addition, we have

$$
\nabla\left(\left|\nabla \Phi^{k}\right|_{\delta} \Delta \Phi^{k+1}\right) /\left|\nabla \Phi^{k}\right|_{\delta}=\nabla \Delta \Phi^{k+1}+\Delta \Phi^{k+1} \frac{\nabla^{2} \Phi^{k} \nabla \Phi^{k}}{\left|\nabla \Phi^{k}\right|_{\delta}^{2}} .
$$

By the face that $\left|\nabla \Phi^{k}\right|_{\delta} \geqslant \delta$ for all $k$, we have

$$
\left|\nabla \mathbf{h}_{\delta}^{k}\right|\left|\nabla \Phi^{k}\right|_{\delta} \leqslant\left|\nabla \Delta \Phi^{k}\right|+\frac{6\left|\nabla^{2} \Phi^{k}\right|^{2}}{\delta}+\left|D^{3} \Phi^{k}\right|
$$

and

$$
\left|\nabla\left(\left|\nabla \Phi^{k}\right|_{\delta} \Delta \Phi^{k+1}\right)\right| /\left|\nabla \Phi^{k}\right|_{\delta} \leqslant\left|\nabla \Delta \Phi^{k+1}\right|+\frac{1}{\delta}\left|\Delta \Phi^{k+1}\right|\left|D^{2} \Phi^{k}\right| .
$$

Consequently

$$
L\left\|\left|\nabla \Phi^{k}\right|_{\delta} \nabla \mathbf{h}_{\delta}^{k}\right\|_{2}^{2} \leqslant C_{1}\left\|D^{3} \Phi^{k}\right\|_{2}^{2}+C_{2}\left\|D^{2} \Phi^{k}\right\|_{4}^{4}
$$

and

$$
\begin{aligned}
\left\|\nabla\left(\left|\nabla \Phi^{k}\right|_{\delta} \Delta \Phi^{k+1}\right) /\left|\nabla \Phi^{k}\right|_{\delta}\right\|_{2}^{2} & \leqslant\left\|\nabla \Delta \Phi^{k+1}\right\|_{2}^{2}+C_{4}\left\|\Delta \Phi^{k+1} \nabla^{2} \Phi^{k}\right\|_{2}^{2} \\
& \leqslant C_{3}\left\|D^{3} \Phi^{k+1}\right\|_{2}^{2}+C_{4}\left\|D^{2} \Phi^{k+1}\right\|_{4}^{4}+C_{4}\left\|D^{2} \Phi^{k}\right\|_{4}^{4} .
\end{aligned}
$$

Therefore, we have the following estimate with lemma 3.3 and 3.4:

$$
L\left\|\left|\nabla \Phi^{k}\right|_{\delta} \nabla \mathbf{h}_{\delta}^{k}\right\|_{2}^{2} \leqslant C_{5}\left\|D^{3} \Phi^{k}\right\|_{2}^{2} \leqslant C_{6}\left\|\nabla \Delta \Phi^{k}\right\|_{2}^{2},
$$

and

$$
\left\|\nabla\left(\left|\nabla \Phi^{k}\right|_{\delta} \Delta \Phi^{k+1}\right) /\left|\nabla \Phi^{k}\right|_{\delta}\right\|_{2}^{2} \leqslant C_{7}\left\|\nabla \Delta \Phi^{k+1}\right\|_{2}^{2}+C_{8}\left\|\nabla \Delta \Phi^{k}\right\|_{2}^{2} .
$$

By plugging into (3.14) we obtain

$$
\left\|\nabla \Phi^{k+1}\right\|_{2}^{2}+\left(\beta-C_{7} \varepsilon\right) d t\left\|\nabla \Delta \Phi^{k+1}\right\|_{2}^{2} \leqslant\left\|\nabla \Phi^{k}\right\|_{2}^{2}+\left(\beta+C_{6}+C_{8} \varepsilon\right) d t\left\|\nabla \Delta \Phi^{k}\right\|_{2}^{2} .
$$

By choosing $\varepsilon=\frac{\beta}{2 C_{7}}$ we obtain

$$
\begin{aligned}
\left\|\nabla \Phi^{k+1}\right\|_{2}^{2}+\frac{\beta}{2} d t\left\|\nabla \Delta \Phi^{k+1}\right\|^{2} & \leqslant\left\|\nabla \Phi^{k}\right\|_{2}^{2}+\left(\beta+C_{6}+\frac{\beta C_{8}}{2 C_{7}}\right) d t\left\|\nabla \Delta \Phi^{k}\right\|^{2} \\
& \leqslant\left(1+\left(1+2 C_{6} / \beta+C_{8} / C_{7}\right) d t\right)\left(\left\|\nabla \Phi^{k}\right\|_{2}^{2}+\frac{\beta}{2} d t\left\|\nabla \Delta \Phi^{k}\right\|^{2}\right) .
\end{aligned}
$$

Taking $K_{1}=\beta / 2$ and $K_{2}=\left(1+2 C_{6} / \beta+C_{8} / C_{7}\right) d t$, then we have the following inequality by induction.

$$
\begin{aligned}
\left\|\nabla \Phi^{k}\right\|_{2}^{2}+K_{1} d t\left\|\nabla \Delta \Phi^{k}\right\|^{2} & \leqslant\left(1+K_{2} d t\right)\left(\left\|\nabla \Phi_{k-1}\right\|_{2}^{2}+K_{1} d t\left\|\nabla \Delta \Phi_{k-1}\right\|^{2}\right) \\
& \leqslant\left(1+K_{2} d t\right)^{k}\left(\left\|\nabla \Phi_{0}\right\|_{2}^{2}+K_{1} d t\left\|\nabla \Delta \Phi_{0}\right\|^{2}\right) \\
& \leqslant e^{K_{2} T}\left(\left\|\nabla \Phi_{0}\right\|_{2}^{2}+K_{1} d t\left\|\nabla \Delta \Phi_{0}\right\|^{2}\right) .
\end{aligned}
$$

which gives the boundedness of the numerical solution.

In this proposition we make the assumption that $\left\|\nabla \Phi^{k}\right\|_{\infty} \leqslant K$. This assumption is reasonable when we are using a level set method for curve evolution problems. We 
should keep the level function smooth to avoid any undesired singulary, thus we always reinitialize the level set function once $\left\|\nabla \Phi^{k}\right\|_{\infty}$ reaches the given upper bound $K$. The assumption $\left\|\nabla \Phi^{k}\right\|_{\infty} \leqslant K$ is used in the proof of convergence. The convergence of the discrete solution to the continuous solution as $d t \rightarrow 0$ is included in the following proposition.

Proposition 3.7. (Convergence) Under the same assumptions as in Theorem 3.1, the discretization error $e^{k}=\phi^{k}-\Phi^{k}$ with $k d t \leqslant T$ for a fixed $T>0$ satisfies

$$
\left\|\nabla e^{k}\right\|_{2}^{2}+K_{1} d t\left\|\nabla \Delta e^{k}\right\|_{2}^{2} \leqslant T e^{K_{2} T} \cdot C d t^{2}
$$

for some constants $C, K_{1}, K_{2}$.

Proof. Subtracting (21) from (22) we obtain

$\frac{e^{k+1}-e^{k}}{d t}+\beta \Delta^{2} e^{k+1}-\beta \Delta^{2} e^{k}=-\left|\nabla \phi^{k}\right|_{\delta} \nabla_{\Gamma}\left(g\left(h_{\delta}^{k}\right) \nabla_{\Gamma} h_{\delta}^{k}\right)+\left|\nabla \Phi^{k}\right|_{\delta} \nabla_{\boldsymbol{\Gamma}}\left(g\left(\mathbf{h}_{\delta}^{k}\right) \nabla_{\Gamma} \mathbf{h}_{\delta}^{k}\right)+\tau^{k}$.

We use the same technique as the proof of proposition 2. Multiplying both sides with $\Delta e^{k+1}$ and integrating by parts, we obtain

$$
\begin{aligned}
& \frac{\left\|\nabla e^{k+1}\right\|_{2}^{2}-\left\langle\nabla e^{k}, \nabla e^{k+1}\right\rangle}{d t}+\beta\left(\left\|\nabla \Delta e^{k+1}\right\|_{2}^{2}-\left\langle\nabla \Delta e^{k+1}, \nabla \Delta e^{k}\right\rangle\right) \\
& =-\left\langle g\left(h_{\delta}^{k}\right) \nabla_{\Gamma} h_{\delta}^{k}, \nabla_{\Gamma}\left(\left|\nabla \phi^{k}\right|_{\delta} \Delta e^{k+1}\right)\right\rangle+\left\langle g\left(\mathbf{h}_{\delta}^{k}\right) \nabla_{\boldsymbol{\Gamma}} \mathbf{h}_{\delta}^{k}, \nabla_{\boldsymbol{\Gamma}}\left(\left|\nabla \Phi^{k}\right|_{\delta} \Delta e^{k+1}\right)\right\rangle \\
& +\left\langle\nabla \Delta^{-1} \tau^{k}, \nabla \Delta e^{k+1}\right\rangle \\
& =\left\langle\nabla \Delta^{-1} \tau^{k}, \nabla \Delta e^{k+1}\right\rangle-\left\langle g\left(h_{\delta}^{k}\right) \nabla_{\Gamma} h_{\delta}^{k}-g\left(\mathbf{h}_{\delta}^{k}\right) \nabla_{\boldsymbol{\Gamma}} \mathbf{h}_{\delta}^{k}, \nabla_{\boldsymbol{\Gamma}}\left(\left|\nabla \Phi^{k}\right|_{\delta} \Delta e^{k+1}\right)\right\rangle \\
& -\left\langle g\left(h_{\delta}^{k}\right) \nabla_{\Gamma} h_{\delta}^{k}, \nabla_{\Gamma}\left(\left|\nabla \phi^{k}\right|_{\delta} \Delta e^{k+1}\right)-\nabla_{\Gamma}\left(\left|\nabla \Phi^{k}\right|_{\delta} \Delta e^{k+1}\right)\right\rangle \text {. }
\end{aligned}
$$

For the difference terms above, we split them into five terms.

$$
\begin{aligned}
& g\left(h_{\delta}^{k}\right) \nabla_{\Gamma} h_{\delta}^{k}-g\left(\mathbf{h}_{\delta}^{k}\right) \nabla_{\boldsymbol{\Gamma}} \mathbf{h}_{\delta}^{k} \\
= & \left(g\left(h_{\delta}^{k}\right)-g\left(\mathbf{h}_{\delta}^{k}\right)\right) \nabla_{\Gamma} h_{\delta}^{k}+g\left(\mathbf{h}_{\delta}^{k}\right)\left(\nabla_{\Gamma}-\nabla_{\boldsymbol{\Gamma}}\right) h_{\delta}^{k}+g\left(\mathbf{h}_{\delta}^{k}\right) \nabla_{\boldsymbol{\Gamma}}\left(h_{\delta}^{k}-\mathbf{h}_{\delta}^{k}\right) \\
= & (I)+(I I)+(I I I), \\
& \nabla_{\Gamma}\left(\left|\nabla \phi^{k}\right|_{\delta} \Delta e^{k+1}\right)-\nabla_{\boldsymbol{\Gamma}}\left(\left|\nabla \Phi^{k}\right|_{\delta} \Delta e^{k+1}\right) \\
= & \left(\nabla_{\Gamma}-\nabla_{\boldsymbol{\Gamma}}\right)\left(\left|\nabla \phi^{k}\right|_{\delta} \Delta e^{k+1}\right)+\nabla_{\boldsymbol{\Gamma}}\left(\left|\nabla \phi^{k}\right|_{\delta} \Delta e^{k+1}-\left|\nabla \Phi^{k}\right|_{\delta} \Delta e^{k+1}\right) \\
= & (I V)+(V) .
\end{aligned}
$$

Now we estimate $(I)-(V)$. First we estimate $h_{\delta}^{k}-\mathbf{h}_{\delta}^{k}$.

$$
\begin{aligned}
h_{\delta}^{k}-\mathbf{h}_{\delta}^{k} & =\left(\frac{\Delta \phi^{k}}{\left|\nabla \phi^{k}\right|_{\delta}}-\frac{\left(\nabla \Phi^{k}\right)^{T} \nabla^{2} \phi^{k} \nabla \phi^{k}}{\left|\nabla \phi^{k}\right|_{\delta}^{3}}\right)-\left(\frac{\Delta \Phi^{k}}{\left|\nabla \Phi^{k}\right|_{\delta}}-\frac{\left(\nabla \Phi^{k}\right)^{T} \nabla^{2} \Phi^{k} \nabla \Phi^{k}}{\left|\nabla \Phi^{k}\right|_{\delta}^{3}}\right) \\
& =\left(\frac{\Delta \phi^{k}}{\left|\nabla \phi^{k}\right|_{\delta}}-\frac{\Delta \Phi^{k}}{\left|\nabla \Phi^{k}\right|_{\delta}}\right)-\left(\frac{\left(\nabla \phi^{k}\right)^{T} \nabla^{2} \phi^{k} \nabla \phi^{k}}{\left|\nabla \phi^{k}\right|_{\delta}^{3}}-\frac{\left(\nabla \Phi^{k}\right)^{T} \nabla^{2} \Phi^{k} \nabla \Phi^{k}}{\left|\nabla \Phi^{k}\right|_{\delta}^{3}}\right)
\end{aligned}
$$

With the fact $|\nabla \phi|_{\delta}>\delta,|\nabla \Phi|_{\delta}>\delta$ and the assumption $\left\|\partial^{\alpha} \phi\right\|_{\infty} \leqslant K$ for $|\alpha| \leqslant 3$, 
$\|\nabla \Phi\|_{\infty} \leqslant K$, we obtain

$$
\begin{aligned}
\left|\frac{\Delta \phi^{k}}{\left|\nabla \phi^{k}\right|_{\delta}}-\frac{\Delta \Phi^{k}}{\left|\nabla \Phi^{k}\right|_{\delta}}\right| & =\left|\frac{\Delta \phi^{k}-\Delta \Phi^{k}}{\left|\nabla \Phi^{k}\right|_{\delta}}+\frac{\Delta \phi^{k}\left(\left|\nabla \Phi^{k}\right|_{\delta}-\left|\nabla \phi^{k}\right|_{\delta}\right)}{\left.\left.\left|\nabla \phi^{k}\right|_{\delta}\right|^{k} \Phi^{k}\right|_{\delta}}\right| \\
& \leqslant \frac{\left|\Delta \phi^{k}-\Delta \Phi^{k}\right|}{\left|\nabla \Phi^{k}\right|_{\delta}}+\frac{\left.\left|\Delta \phi^{k}\right||| \nabla \Phi^{k}\right|_{\delta}-\left|\nabla \Phi^{k}\right|_{\delta} \mid}{\left|\nabla \phi^{k}\right|_{\delta}\left|\nabla \Phi^{k}\right|_{\delta}} \\
& \leqslant \frac{\left|\Delta e^{k}\right|}{\left|\nabla \Phi^{k}\right|_{\delta}}+\frac{\left|\Delta \phi^{k}\right|\left|\nabla e^{k}\right|}{\left|\nabla \Phi^{k}\right|_{\delta}\left|\nabla \Phi^{k}\right|_{\delta}} \\
& \leqslant \frac{\left|\Delta e^{k}\right|}{\delta}+\frac{\left|\nabla e^{k}\right|\left|D^{2} \phi^{k}\right|}{\delta^{2}} .
\end{aligned}
$$

and

$$
\begin{gathered}
\left|\frac{\left(\nabla \phi^{k}\right)^{T} \nabla^{2} \phi^{k} \nabla \phi^{k}}{\left|\nabla \phi^{k}\right|_{\delta}^{3}}-\frac{\left(\nabla \Phi^{k}\right)^{T} \nabla^{2} \Phi^{k} \nabla \Phi^{k}}{\left|\nabla \Phi^{k}\right|_{\delta}^{3}}\right| \\
\leqslant\left|\frac{\left(\nabla \phi^{k}\right)^{T} \nabla^{2} \phi^{k} \nabla \phi^{k}}{\left|\nabla \Phi^{k}\right|_{\delta}^{3}}-\frac{\left(\nabla \Phi^{k}\right)^{T} \nabla^{2} \Phi^{k} \nabla \Phi^{k}}{\left|\nabla \Phi^{k}\right|_{\delta}^{3}}\right|+\left|\frac{\left(\nabla \phi^{k}\right)^{T} \nabla^{2} \phi^{k} \nabla \phi^{k}}{\left|\nabla \phi^{k}\right|_{\delta}^{3}}-\frac{\left(\nabla \phi^{k}\right)^{T} \nabla^{2} \phi^{k} \nabla \phi^{k}}{\left|\nabla \Phi^{k}\right|_{\delta}^{3}}\right| \\
\leqslant\left|\frac{\left(\nabla \phi^{k}-\nabla \Phi^{k}\right)^{T} \nabla^{2} \phi^{k} \nabla \phi^{k}}{\left|\nabla \Phi^{k}\right|_{\delta}^{3}}\right|+\left|\frac{\left(\nabla \Phi^{k}\right)^{T} \nabla^{2} \phi^{k}\left(\nabla \phi^{k}-\nabla \Phi^{k}\right)}{\left|\nabla \Phi^{k}\right|_{\delta}^{3}}\right|+\left|\frac{\left(\nabla \Phi^{k}\right)^{T}\left(\nabla^{2} \phi^{k}-\nabla^{2} \Phi^{k}\right) \nabla \phi^{k}}{\left|\nabla \Phi^{k}\right|_{\delta}^{3}}\right| \\
+\left|\left(\nabla \phi^{k}\right)^{T} \nabla^{2} \phi^{k} \nabla \phi^{k}\right|\left|\frac{\left(\left|\nabla \phi^{k}\right|_{\delta}-\left|\nabla \Phi^{k}\right|_{\delta}\right)\left(\left|\nabla \phi^{k}\right|_{\delta}^{2}+\left|\nabla \phi^{k}\right|_{\delta}\left|\nabla \Phi^{k}\right|_{\delta}+\left|\nabla \Phi^{k}\right|_{\delta}^{2}\right)}{\left|\nabla \phi^{k}\right|_{\delta}^{3}\left|\nabla \Phi^{k}\right|_{\delta}^{3}}\right| \\
\leqslant\left|\frac{\left(\nabla e^{k}\right)^{T} \nabla^{2} \phi^{k} \nabla \phi^{k}}{\left|\nabla \Phi^{k}\right|_{\delta}^{3}}\right|+\left|\frac{\left(\nabla \Phi^{k}\right)^{T} \nabla^{2} \phi^{k} \nabla e^{k}}{\left|\nabla \Phi^{k}\right|_{\delta}^{3}}\right|+\left|\frac{\left(\nabla \Phi^{k}\right)^{T} \nabla^{2} e^{k} \nabla \phi^{k}}{\left|\nabla \Phi^{k}\right|_{\delta}^{3}}\right| \\
+\left|\left(\nabla \phi^{k}\right)^{T} \nabla^{2} \phi^{k} \nabla \phi^{k}\right| \mid \frac{\left(\left|\nabla e^{k}\right|_{\delta}\right)\left(\left|\nabla \phi^{k}\right|_{\delta}^{2}+\left|\nabla \phi^{k}\right|_{\delta}\left|\nabla \Phi^{k}\right|_{\delta}+\left|\nabla \Phi^{k}\right|_{\delta}^{2}\right)}{\left.\left.\left|\nabla \phi^{k}\right|_{\delta}^{3}\right|^{k} \Phi_{\delta}^{k}\right|_{\delta} ^{3}} \\
\leqslant C\left(\left|\Delta e^{k}\right|+\left|\nabla e^{k}\right|\right) .
\end{gathered}
$$

Therefore, we have the following inequalities.

$$
\left|h_{\delta}^{k}-\mathbf{h}_{\delta}^{k}\right| \leqslant C_{1}\left(\left|\Delta e^{k}\right|+\left|\nabla e^{k}\right|\right) .
$$

and

$$
\begin{aligned}
\left|g\left(h_{\delta}^{k}\right)-g\left(\mathbf{h}_{\delta}^{k}\right)\right| & =\left|g^{\prime}\left(h^{*}\right)\left(h_{\delta}^{k}-\mathbf{h}_{\delta}^{k}\right)\right| \\
& \leqslant L C_{1}\left(\left|\Delta e^{k}\right|+\left|\nabla e^{k}\right|\right) .
\end{aligned}
$$

and

$$
\begin{aligned}
\left|\left(\nabla_{\Gamma}-\nabla_{\boldsymbol{\Gamma}}\right) h_{\delta}^{k}\right| & =\left|\left(\left(I-\frac{\nabla \phi^{k} \nabla\left(\phi^{k}\right)^{T}}{\left|\nabla \phi^{k}\right|_{\delta}^{2}}\right)-\left(I-\frac{\nabla \Phi^{k} \nabla\left(\Phi^{k}\right)^{T}}{\left|\nabla \Phi^{k}\right|_{\delta}^{2}}\right)\right) \nabla h_{\delta}^{k}\right| \\
& =\left|\left(\frac{\nabla \phi^{k} \nabla\left(\phi^{k}\right)^{T}}{\left|\nabla \phi^{k}\right|_{\delta}^{2}}-\frac{\nabla \Phi^{k} \nabla\left(\Phi^{k}\right)^{T}}{\left|\nabla \Phi^{k}\right|_{\delta}^{2}}\right) \nabla h_{\delta}^{k}\right| \\
& \leqslant C_{2}\left|\nabla e^{k}\right| .
\end{aligned}
$$

To estimate $\left|\nabla\left(h_{\delta}^{k}-\mathbf{h}_{\delta}^{k}\right)\right|$, recall that we represent $\nabla \mathbf{h}_{\delta}$ by

$$
\begin{aligned}
\nabla \mathbf{h}_{\delta} & =\frac{\nabla \Delta \Phi^{k}}{\left|\nabla \Phi^{k}\right|_{\delta}}-\frac{\Delta \Phi^{k} \nabla^{2} \Phi^{k} \nabla \Phi^{k}}{\left|\nabla \Phi^{k}\right|_{\delta}^{3}}-\frac{2 \nabla^{2} \Phi^{k} \nabla^{2} \Phi^{k} \nabla \Phi^{k}}{\left|\nabla \Phi^{k}\right|_{\delta}^{3}} \\
& -\frac{\nabla \Phi^{k} \nabla\left(\nabla^{2} \Phi^{k}\right) \nabla \Phi^{k}}{\left|\nabla \Phi^{k}\right|_{\delta}^{3}}+\frac{3\left(\nabla \Phi^{k}\right)^{T} \nabla^{2} \Phi^{k} \nabla \Phi^{k} \nabla^{2} \Phi^{k} \nabla \Phi^{k}}{\left|\nabla \Phi^{k}\right|_{\delta}^{5}} .
\end{aligned}
$$


and we have the corresponding one for $\nabla h_{\delta}^{k}$. Then the estimate goes as follows.

$$
\begin{aligned}
\left|\nabla\left(h_{\delta}^{k}-\mathbf{h}_{\delta}^{k}\right)\right| & \leqslant\left|\frac{\nabla \Delta \Phi^{k}}{\left|\nabla \Phi^{k}\right|_{\delta}}-\frac{\nabla \Delta \phi^{k}}{\left|\nabla \phi^{k}\right|_{\delta}}\right|+\left|\frac{\Delta \Phi^{k} \nabla^{2} \Phi^{k} \nabla \Phi^{k}}{\left|\nabla \Phi^{k}\right|_{\delta}^{3}}-\frac{\Delta \phi^{k} \nabla^{2} \phi^{k} \nabla \phi^{k}}{\left|\nabla \phi^{k}\right|_{\delta}^{3}}\right| \\
+2 \mid & \frac{\nabla^{2} \Phi^{k} \nabla^{2} \Phi^{k} \nabla \Phi^{k}}{\left|\nabla \Phi^{k}\right|_{\delta}^{3}}-\frac{\nabla^{2} \Phi^{k} \nabla^{2} \Phi^{k} \nabla \Phi^{k}}{\left|\nabla \Phi^{k}\right|_{\delta}^{3}}|+| \frac{\nabla \Phi^{k} \nabla\left(\nabla^{2} \Phi^{k}\right) \nabla \Phi^{k}}{\left|\nabla \Phi^{k}\right|_{\delta}^{3}}-\frac{\nabla \phi^{k} \nabla\left(\nabla^{2} \phi^{k}\right) \nabla \phi^{k}}{\left|\nabla \phi^{k}\right|_{\delta}^{3}} \mid \\
& +3\left|\frac{\left(\nabla \Phi^{k}\right)^{T} \nabla^{2} \Phi^{k} \nabla \Phi^{k} \nabla^{2} \Phi^{k} \nabla \Phi^{k}}{\left|\nabla \Phi^{k}\right|_{\delta}^{5}}-\frac{\left(\nabla \phi^{k}\right)^{T} \nabla^{2} \phi^{k} \nabla \phi^{k} \nabla^{2} \phi^{k} \nabla \phi^{k}}{\left|\nabla \phi^{k}\right|_{\delta}^{5}}\right| .
\end{aligned}
$$

With similar analysis as above, we have

$$
\left|\frac{\nabla \Delta \Phi^{k}}{\left|\nabla \Phi^{k}\right|_{\delta}}-\frac{\nabla \Delta \phi^{k}}{\left|\nabla \phi^{k}\right|_{\delta}}\right| \leqslant C\left(\left|\nabla \Delta e^{k}\right|+\left|\nabla e^{k}\right|\right)
$$

and

$$
\left|\frac{\nabla \Phi^{k} \nabla\left(\nabla^{2} \Phi^{k}\right) \nabla \Phi^{k}}{\left|\nabla \Phi^{k}\right|_{\delta}^{3}}-\frac{\nabla \phi^{k} \nabla\left(\nabla^{2} \phi^{k}\right) \nabla \phi^{k}}{\left|\nabla \phi^{k}\right|_{\delta}^{3}}\right| \leqslant C\left(\left|D^{3} e^{k}\right|+\left|\nabla e^{k}\right|\right) .
$$

In addition, we use the fact that $\Delta \Phi^{k}=\Delta \phi^{k}-\Delta e^{k}$ and $\nabla^{2} \Phi^{k}=\nabla^{2} \phi^{k}-\nabla^{2} e^{k}$ to estimate the other three difference terms. Here we use again the assumption that the derivatives of $\phi$ is bounded.

$$
\begin{aligned}
\Delta \Phi^{k} \nabla^{2} \Phi^{k} & =\left(\Delta \phi^{k}-\Delta e^{k}\right)\left(\nabla^{2} \phi^{k}-\nabla^{2} e^{k}\right) \\
& =\Delta \phi^{k} \nabla^{2} \phi^{k}-\Delta \phi^{k} \nabla^{2} e^{k}-\Delta e^{k} \nabla^{2} \phi^{k}+\Delta e^{k} \nabla^{2} e^{k} .
\end{aligned}
$$

Therefore, we obtain the following estimate.

$$
\begin{aligned}
& \left|\frac{\Delta \Phi^{k} \nabla^{2} \Phi^{k} \nabla \Phi^{k}}{\left|\nabla \Phi^{k}\right|_{\delta}^{3}}-\frac{\Delta \phi^{k} \nabla^{2} \phi^{k} \nabla \phi^{k}}{\left|\nabla \phi^{k}\right|_{\delta}^{3}}\right| \\
\leqslant & \left|\frac{\left(\Delta \Phi^{k} \nabla^{2} \Phi^{k}-\Delta \phi^{k} \nabla^{2} \phi^{k}\right) \nabla \Phi^{k}}{\left|\nabla \Phi^{k}\right|_{\delta}^{3}}\right|+\left|\frac{\Delta \phi^{k} \nabla^{2} \phi^{k}\left(\nabla \Phi^{k}-\nabla \phi^{k}\right)}{\left|\nabla \Phi^{k}\right|_{\delta}^{3}}\right| \\
& \quad+\left|\frac{\Delta \phi^{k} \nabla^{2} \phi^{k} \nabla \phi^{k}}{\left|\nabla \Phi^{k}\right|_{\delta}^{3}}-\frac{\Delta \phi^{k} \nabla^{2} \phi^{k} \nabla \phi^{k}}{\left|\nabla \phi^{k}\right|_{\delta}^{3}}\right| \\
\leqslant & C\left(\left|D^{2} e^{k}\right|^{2}+\left|D^{2} e^{k}\right|+\left|\nabla e^{k}\right|\right) .
\end{aligned}
$$

Similarly we have

$$
\left|\frac{\nabla^{2} \Phi^{k} \nabla^{2} \Phi^{k} \nabla \Phi^{k}}{\left|\nabla \Phi^{k}\right|_{\delta}^{3}}-\frac{\nabla^{2} \Phi^{k} \nabla^{2} \Phi^{k} \nabla \Phi^{k}}{\left|\nabla \Phi^{k}\right|_{\delta}^{3}}\right| \leqslant C\left(\left|D^{2} e^{k}\right|^{2}+\left|D^{2} e^{k}\right|+\left|\nabla e^{k}\right|\right) .
$$

and

$\left|\frac{\left(\nabla \Phi^{k}\right)^{T} \nabla^{2} \Phi^{k} \nabla \Phi^{k} \nabla^{2} \Phi^{k} \nabla \Phi^{k}}{\left|\nabla \Phi^{k}\right|_{\delta}^{5}}-\frac{\left(\nabla \phi^{k}\right)^{T} \nabla^{2} \phi^{k} \nabla \phi^{k} \nabla^{2} \phi^{k} \nabla \phi^{k}}{\left|\nabla \phi^{k}\right|_{\delta}^{5}}\right| \leqslant C\left(\left|D^{2} e^{k}\right|^{2}+\left|D^{2} e^{k}\right|+\left|\nabla e^{k}\right|\right)$.

Thus we obtain the following estimate

$$
\left|\nabla\left(h_{\delta}^{k}-\mathbf{h}_{\delta}^{k}\right)\right| \leqslant C_{3}\left(\left|D^{3} e^{k}\right|+\left|D^{2} e^{k}\right|+\left|D^{2} e^{k}\right|^{2}+\left|\nabla e^{k}\right|\right) .
$$

Consequently the estimation for $(I),(I I),(I I I)$ are

$$
|(I)| \leqslant\left|g\left(h_{\delta}^{k}\right)-g\left(\mathbf{h}_{\delta}^{k}\right)\right|\left|\nabla h_{\delta}^{k}\right| \leqslant C_{4}\left(\left|\Delta e^{k}\right|+\left|\nabla e^{k}\right|\right),
$$




$$
\begin{gathered}
|(I I)|=\left|g\left(\mathbf{h}_{\delta}^{k}\right)\left(\nabla_{\Gamma}-\nabla_{\boldsymbol{\Gamma}}\right) h_{\delta}^{k}\right| \leqslant L C_{1}\left|\left(\nabla_{\Gamma}-\nabla_{\boldsymbol{\Gamma}}\right) h_{\delta}^{k}\right| \leqslant C_{5}\left|\nabla e^{k}\right|, \\
|(I I I)|=\left|g\left(\mathbf{h}_{\delta}^{k}\right) \nabla_{\boldsymbol{\Gamma}}\left(h_{\delta}^{k}-\mathbf{h}_{\delta}^{k}\right)\right| \leqslant L\left|\nabla\left(h_{\delta}^{k}-\mathbf{h}_{\delta}^{k}\right)\right| \\
\leqslant C_{6}\left(\left|D^{3} e^{k}\right|+\left|D^{2} e^{k}\right|+\left|D^{2} e^{k}\right|^{2}+\left|\nabla e^{k}\right|\right) .
\end{gathered}
$$

Estimating $(I V)$ and $(V)$ is slightly different. We have

$$
\nabla\left(\left|\nabla \phi^{k}\right|_{\delta} \Delta e^{k+1}\right)=\frac{\nabla \Delta e^{k+1}}{\left|\nabla \phi^{k}\right|_{\delta}}+\Delta e^{k+1} \frac{\nabla^{2} \phi^{k} \nabla \phi^{k}}{\left|\nabla \phi^{k}\right|^{3}} .
$$

Therefore

$$
\begin{aligned}
|(I V)| & =\left|\left(\nabla_{\Gamma}-\nabla_{\boldsymbol{\Gamma}}\right)\left(\left|\nabla \phi^{k}\right|_{\delta} \Delta e^{k+1}\right)\right| \\
& =\left|\left(\frac{\nabla \phi^{k} \nabla\left(\phi^{k}\right)^{T}}{\left|\nabla \phi^{k}\right|_{\delta}^{2}}-\frac{\nabla \Phi^{k} \nabla\left(\Phi^{k}\right)^{T}}{\left|\nabla \Phi^{k}\right|_{\delta}^{2}}\right) \nabla\left(\left|\nabla \phi^{k}\right|_{\delta} \Delta e^{k+1}\right)\right| \\
& \leqslant C_{7}\left|\nabla e^{k}\right|\left|\nabla\left(\left|\nabla \phi^{k}\right|_{\delta} \Delta e^{k+1}\right)\right| \\
& \leqslant C_{8}\left|\nabla e^{k}\right|\left(\left|D^{3} e^{k+1}\right|+\left|D^{2} e^{k+1}\right|\right), \\
|(V)| & =\left|\nabla_{\boldsymbol{\Gamma}}\left(\left|\nabla \phi^{k}\right|_{\delta} \Delta e^{k+1}-\left|\nabla \Phi^{k}\right|_{\delta} \Delta e^{k+1}\right)\right| \\
& \leqslant\left|\nabla\left(\left|\nabla \phi^{k}\right|_{\delta} \Delta e^{k+1}-\left|\nabla \Phi^{k}\right|_{\delta} \Delta e^{k+1}\right)\right| \\
& \leqslant\left.\left|\nabla \Delta e^{k+1}\right||| \nabla \phi^{k}\right|_{\delta}-\left|\nabla \Phi^{k}\right|_{\delta}|+| \Delta e^{k+1}|| \nabla\left(\left|\nabla \phi^{k}\right|_{\delta}-\left|\nabla \Phi^{k}\right|_{\delta}\right) \mid \\
& \leqslant C_{9}\left(\left|D^{3} e^{k+1}\right|\left|\nabla e^{k}\right|+\left|D^{2} e^{k+1}\right|\left|D^{2} e^{k}\right|+\left|D^{2} e^{k+1}\right|\left|\nabla e^{k}\right|\right) .
\end{aligned}
$$

In addition, we use $\nabla^{2} \Phi^{k}=\nabla^{2} \phi^{k}-\nabla^{2} e^{k}$ again and obtain the following estimate

$$
\begin{aligned}
\left|\nabla_{\boldsymbol{\Gamma}}\left(\left|\nabla \Phi^{k}\right|_{\delta} \Delta e^{k+1}\right)\right| & \leqslant\left|\nabla\left(\left|\nabla \Phi^{k}\right|_{\delta} \Delta e^{k+1}\right)\right| \\
& \leqslant\left|\nabla \Phi^{k}\right|_{\delta}\left|\nabla \Delta e^{k}\right|+\left|\Delta e^{k}\right| \frac{\left|\nabla^{2} \Phi^{k} \nabla \Phi^{k}\right|}{\left|\nabla \Phi^{k}\right|_{\delta}^{2}} \\
& \leqslant C_{10}\left(\left|D^{3} e^{k+1}\right|+\left|D^{2} e^{k+1}\right|+\left|D^{2} e^{k+1}\right|\left|D^{2} e^{k}\right|\right) \\
& \leqslant C_{10}\left(\left|D^{3} e^{k+1}\right|+\left|D^{2} e^{k+1}\right|+\left|D^{2} e^{k+1}\right|^{2}+\left|D^{2} e^{k}\right|^{2}\right) .
\end{aligned}
$$

Thus we obtain the following estimate

$$
\begin{aligned}
\left|g\left(h_{\delta}^{k}\right) \nabla_{\Gamma} h_{\delta}^{k}-g\left(\mathbf{h}_{\delta}^{k}\right) \nabla_{\boldsymbol{\Gamma}} \mathbf{h}_{\delta}^{k}\right| & \leqslant C_{11}\left(\left|D^{3} e^{k}\right|+\left|D^{2} e^{k}\right|+\left|D^{2} e^{k}\right|^{2}+\left|\nabla e^{k}\right|\right) \\
\left|\nabla_{\Gamma}\left(\left|\nabla \phi^{k}\right|_{\delta} \Delta e^{k+1}\right)-\nabla_{\boldsymbol{\Gamma}}\left(\left|\nabla \Phi^{k}\right|_{\delta} \Delta e^{k+1}\right)\right| & \leqslant C_{12}\left(\left|D^{3} e^{k+1}\right|\left|\nabla e^{k}\right|+\left|D^{2} e^{k+1}\right|\left|D^{2} e^{k}\right|+\left|D^{2} e^{k+1}\right|\left|\nabla e^{k}\right|\right) \\
& \leqslant C_{12}\left(\left|D^{3} e^{k+1}\right|+\left|D^{2} e^{k+1}\right|\right)\left(\left|D^{2} e^{k}\right|+\left|\nabla e^{k}\right|\right) .
\end{aligned}
$$

Consequently applying lemma 3.3 and 3.4 , and the fact $\left|\nabla e^{k}\right| \leqslant\left|\nabla \phi^{k}\right|+\left|\nabla \Phi^{k}\right| \leqslant$ $2 K$, we obtain

$$
\begin{aligned}
& -\left\langle g\left(h_{\delta}^{k}\right) \nabla_{\Gamma} h_{\delta}^{k}-g\left(\mathbf{h}_{\delta}^{k}\right) \nabla_{\boldsymbol{\Gamma}} \mathbf{h}_{\delta}^{k}, \nabla_{\boldsymbol{\Gamma}}\left(\left|\nabla \Phi^{k}\right|_{\delta} \Delta e^{k+1}\right)\right\rangle \\
\leqslant & \left\langle\left|g\left(h_{\delta}^{k}\right) \nabla_{\Gamma} h_{\delta}^{k}-g\left(\mathbf{h}_{\delta}^{k}\right) \nabla_{\boldsymbol{\Gamma}} \mathbf{h}_{\delta}^{k}\right|,\left|\nabla_{\boldsymbol{\Gamma}}\left(\left|\nabla \Phi^{k}\right|_{\delta} \Delta e^{k+1}\right)\right|\right\rangle \\
\leqslant & C_{13}\left\langle\left|D^{3} e^{k+1}\right|+\left|D^{2} e^{k+1}\right|+\left|D^{2} e^{k+1}\right|^{2}+\left|D^{2} e^{k}\right|^{2},\left|D^{3} e^{k}\right|+\left|D^{2} e^{k}\right|+\left|D^{2} e^{k}\right|+\left|\nabla e^{k}\right|\right\rangle \\
\leqslant & C_{13} \varepsilon\left(\left\|D^{3} e^{k+1}\right\|_{2}^{2}+\left|D^{2} e^{k+1}\right|_{2}^{2}+\left|D^{2} e^{k+1}\right|_{4}^{4}+\left|D^{2} e^{k}\right|_{4}^{4}\right) \\
& \quad+C_{13} / \varepsilon\left(\left|D^{3} e^{k}\right|_{2}^{2}+\left|D^{2} e^{k}\right|_{2}^{2}+\left|D^{3} e^{k}\right|_{4}^{4}+\left|\nabla e^{k}\right|_{2}^{2}\right) \\
\leqslant & C_{14} \varepsilon\left(\left\|D^{3} e^{k+1}\right\|_{2}^{2}+\left|\nabla e^{k+1}\right|_{2}^{2}+|| D^{3} e^{k} \|_{2}^{2}+\left|\nabla e^{k}\right|_{2}^{2}\right)+C_{14} / \varepsilon\left(\left|D^{3} e^{k}\right|_{2}^{2}+\left|\nabla e^{k}\right|_{2}^{2}\right) \\
= & C_{14} \varepsilon\left(\left\|D^{3} e^{k+1}\right\|_{2}^{2}+\left|\nabla e^{k+1}\right|_{2}^{2}\right)+\left(C_{14} \varepsilon+C_{14} / \varepsilon\right)\left(\left|D^{3} e^{k}\right|_{2}^{2}+\left|\nabla e^{k}\right|_{2}^{2}\right) .
\end{aligned}
$$


and similarly

$$
\begin{gathered}
\quad\left|\left(g\left(h_{\delta}^{k}\right) \nabla_{\Gamma} h_{\delta}^{k}, \nabla_{\Gamma}\left(\left|\nabla \phi^{k}\right|_{\delta} \Delta e^{k+1}\right)-\nabla_{\boldsymbol{\Gamma}}\left(\left|\nabla \Phi^{k}\right|_{\delta} \Delta e^{k+1}\right)\right)\right| \\
\leqslant C_{15} \varepsilon\left(\left\|D^{3} e^{k+1}\right\|_{2}^{2}+\left|\nabla e^{k+1}\right|_{2}^{2}\right)+C_{15} / \varepsilon\left(\left|D^{3} e^{k}\right|_{2}^{2}+\left|\nabla e^{k}\right|_{2}^{2}\right) .
\end{gathered}
$$

Now we come to the following estimate

$$
\begin{aligned}
& \frac{\left\|\nabla e^{k+1}\right\|_{2}^{2}-\left\langle\nabla e^{k}, \nabla e^{k+1}\right\rangle}{d t}+\beta\left(\left\|\nabla \Delta e^{k+1}\right\|_{2}^{2}-\left\langle\nabla \Delta e^{k+1}, \nabla \Delta e^{k}\right\rangle\right) \\
\leqslant & C_{16} \varepsilon\left(\left\|\nabla e^{k+1}\right\|_{2}^{2}+\left\|\nabla \Delta e^{k+1}\right\|_{2}^{2}\right)+\left(C_{16} \varepsilon+C_{16} / \varepsilon\right)\left(\left\|\nabla e^{k}\right\|_{2}^{2}+\left\|\nabla \Delta e^{k}\right\|_{2}^{2}\right)+\frac{1}{\varepsilon}\left\|\tau^{k}\right\|_{-1}^{2} .
\end{aligned}
$$

where $\varepsilon$ is a arbitrary constant. Applying Cauchy's inequality to the left hand side we obtain

$$
\begin{aligned}
& \left(1-C_{16} \varepsilon d t\right)\left\|\nabla e^{k+1}\right\|_{2}^{2}+\left(\beta-C_{16} \varepsilon\right) d t\left\|\nabla \Delta e^{k+1}\right\|_{2}^{2} \\
\leqslant & \left(1+C_{16} / \varepsilon d t\right)\left\|\nabla e^{k}\right\|_{2}^{2}+\left(\beta+C_{16} \varepsilon+C_{16} / \varepsilon\right) d t\left\|\nabla \Delta e^{k}\right\|_{2}^{2}+1 / \varepsilon\left\|\tau^{k}\right\|_{-1}^{2} .
\end{aligned}
$$

We take $\varepsilon=\frac{\beta}{2 C_{16}}$. Then we can take $K_{1}=1+\beta$ and $K_{2}=\beta+C_{16} \varepsilon+C_{16} / \varepsilon$, as long as $d t$ is small enough, we have

$$
\left\|\nabla e^{k+1}\right\|_{2}^{2}+K_{1} d t\left\|\nabla \Delta e^{k+1}\right\|_{2}^{2} \leqslant\left(1+K_{2} d t\right)\left(\left\|\nabla e^{k}\right\|_{2}^{2}+K_{1} d t\left\|\nabla \Delta e^{k}\right\|_{2}^{2}\right)+\frac{\beta}{C_{10}} d t\left\|\tau^{k}\right\|_{-1}^{2} .
$$

By induction we obtain the following estimate.

$$
\left\|\nabla e^{k}\right\|_{2}^{2}+K_{1} d t\left\|\nabla \Delta e^{k}\right\|_{2}^{2} \leqslant T e^{K_{2} T} \cdot C d t^{2} .
$$

We can see that $\left\|\nabla e^{k}\right\|_{2}$ converges with first order in time.

When using a level set method for curve evolution problems, the existence of corners break the smoothness of the level set function. However, as in the previous discussion, this corner preserving model generalizes the LCIS equation and the corners are in the infinitesimal sense. In Bertozzi and Greer [3] it has been proved in one dimension that the solutions of LCIS equations are smooth and never develop corners in finite time. We believe that it is also true for our model, as long as the curve has no self-intersections, although this has not been proven. In addition, the level set function is smooth in a small neighborhood of the curve if the curve is smooth. However, in numerical implementation, the width of the narrow band to keep the level set function smooth may be very small, consequently $d t$ has to be chosen small enough. When applying the semi-implicit scheme, the numerical experiments shows that we can take $d t \sim d x^{2}$. This is still a great improvement comparing with $d t \sim d x^{4}$ for explicit schemes. Note that the operator $\left(1+d t \cdot \beta \Delta^{2}\right)^{-1}$ is positive definite, it works as a smoothing operator. Empirically we choose the parameter $\beta=1 / 2$ as in $[26,29]$.

In addition, our goal is to evolve the curve, which only involves a small neighborhood of the zero level set, so we can only compute corner preserving term of equation (2.7) in a narrow band around the zero level set of $\phi$ and set it to be zero in other places. This can save computational time. We may reinitialize $\phi$ to be the signed distance function, but not necessarily. According to our analysis, we impose an upper bound $K$ for $\left|\nabla \Phi^{k}\right|$. If $\left|\nabla \Phi^{k}\right|$ exceeds $K$, reinitialization is required.

In two dimension images the operator $\left(1+d t \cdot \beta \Delta^{2}\right)^{-1}$ can be computed using Fast Fourier Transform (FFT) very easily and efficiently. The convergence is still somewhat 
slow when comparing with Chan-Vese model due to the time step restriction. The Chan-Vese model only requires $d t \sim d x$ while our method requires $d t \sim d x^{2}$. In fact we can take advantage of fast methods for Chan-Vese by first solving Chan-Vese to steady state and using this as the initial guess for our method. Since we start with a initial guess that is close to the final result, the reinitialization process during the level set evolution is optional.

The full algorithm is:

\begin{tabular}{|c|c|}
\hline ep 0. & Solve Chan-Vese model and obtain the steady state $\phi$. \\
\hline & Initialize the level set function $\phi$ to the signed distance function. \\
\hline Step 2 . & $\begin{array}{l}\text { Compute } c_{1} \text { and } c_{2} \text { for equation (2.7) and the Chan-Vese energy } \\
\text { term. }\end{array}$ \\
\hline Step 3. & $\begin{array}{l}\text { Compute the corner preserving term in a narrow band around } \\
\text { the zero level set of } \phi \text { and set it to be } 0 \text { in other places. Usually } \\
\text { we choose the narrow band as points within } 3 \text { or } 4 \text { grid size to } \\
\text { the zero level set. }\end{array}$ \\
\hline Step 4. & Update $\phi$ with equation $(2.7)$ \\
\hline Step 5 . & $\begin{array}{l}\text { Reinitialize the level set function } \phi \text { to be the signed distance } \\
\text { function if }\left|\nabla \Phi^{k}\right| \text { exceeds } K \text {. Repeat step } 2 \text { until convergence. }\end{array}$ \\
\hline
\end{tabular}

4. Numerical Results. In this section we show some numerical results for image segmentation with the equation (2.7). Although we employ semi-implicit schemes, the time step is still small for accuracy. Usually we take the order $d t \sim d x^{2}$. For faster convergence, we do not directly solve equation (2.7) with a random initialization, but we start from the steady state of Chan-Vese method and then solve equation (2.7). The time step for equation (2.7) is chosen as $d t=.1 d x^{2}$, while time step for preprocessing with Chan-Vese method is $d t=.1 d x$. As for the computational time, the regular Chan-Vese method takes 2 seconds and our methods takes 21 seconds for the building image of size $128 \times 110$ using $\mathrm{C}++$. For the hyperspectral image of size $100 \times 80$ below, the Chan-Vese method takes 4 seconds and our methods takes 35 seconds.

Figure (4.1) shows the segmentation of a simple shape. (I) is the originally image. (II) shows the segmentation with equation (2.7) and (III) shows the segmentation without corner preserving term. Since the noise is strong in this image, the length regularization term has to be chosen large to avoid the local minima and small noisy pieces. We can see the corners are much better kept with the corner preserving term.

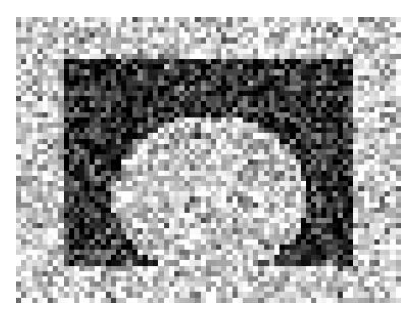

(I) Initial image

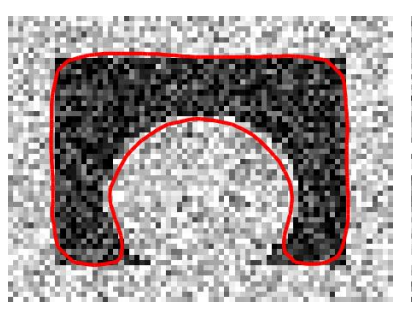

(II) Segmentation without corner (III) Segmentation with corner

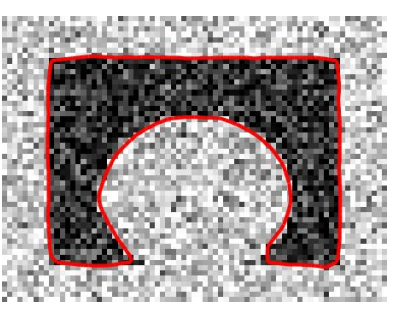

FIG. 4.1. Comparison of Segmentation that with and without corner term on a simple shape.

Figure (4.2) shows the segmentation of a building from Google map. This is a 3 -band color image. To avoid detecting the pieces on the roof, we have to use strong 
regularization. The segmentation with equation (2.7) is better than the segmentation without corner preserving term. And we also see that the two pieces enclosed by the building are also captured by the level set based segmentation method.
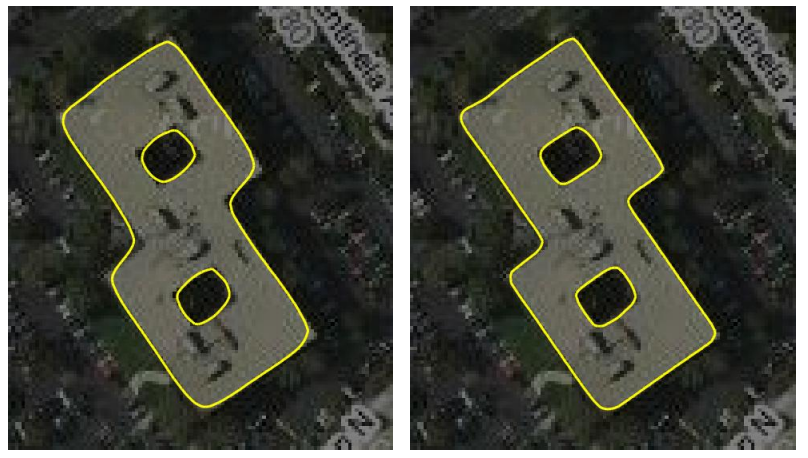

(I) Segmentation without corner (II) Segmentation with corner

FIG. 4.2. Comparison of Segmentation that with and without corner term on a building.

Figure (4.3) shows the segmentation of a Walmart building from hyperspectral image with 163 bands. We can see that the approach also works for hyperspectral image. The dirt near the building can not be separated due to the limitation of the Chan-Vese method, which always separates the 'bright' part from the 'dark' part. Compared with the parking lot, the dirt is obviously bright.
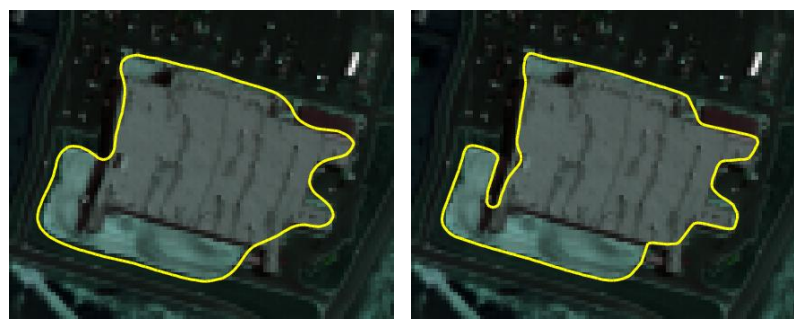

(I) Segmentation without corner (II) Segmentation with corner

FIG. 4.3. Comparison of Segmentation that with and without corner term on a building.

5. Conclusion. In this paper we propose a modification of the Chan-Vese model. Motivated by the low curvature image simplifier, we add a corner preserving term to the Chan-Vese model following a method developed in Droske and Bertozzi [17] for image snakes. With the new model we can capture the sharp corners in the image while we can still manage the complex topology. To solve the high order nonlinear equation, we employ the numerical technique of adding a bilaplacian term and using semi-implicit schemes, which improves the time step from $d t \sim d x^{4}$ to $d t \sim d x^{2}$. We also prove the stability and convergence of the semi-implicit time stepping scheme. We validate our model by numerical tests on color and hyperspectral images. The numerical results also show that this new model is robust to noise. One issue is that due to the nonlinearity and high order, we have to use smaller time steps when comparing with the original Chan-Vese model. Future work could involve faster numerical schemes to speed up this method, or the application of this model to surface representation and reconstruction as in [17]. 
Acknowledgements. We thank Jian Ye and Stanley Osher for useful conversations on the fast algorithm for Chan-Vese model.

\section{REFERENCES}

[1] G. Bellettini and M. Paolini, Anisotropic motion by mean curvature in the context of Finsler geomety", QUADERNO, Hokkaido Math. J., 25 (1996), pp. 537-566.

[2] M. Bertalmio and L.T. Cheng and G. Sapiro and S. Osher, Variational problems and partial differential equations on implicit surfaces, J. Comput. Phys., 174 (2001), pp. 759780.

[3] A. Bertozzi And J.B. Greer, Low curvature image simplifiers: global regularity of smooth solutions and laplacian limiting schemes, Comm. Pure Appl. Math., 57 (2004), pp. 764790.

[4] A. Bertozzi And N. Ju And H. Lu, A biharmonic modified forward time stepping method for fourth order nonlinear diffusion equations, submitted to DCDS.

[5] M. Burger, Numerical simulation of anisotropic surface diffusion with curvature-dependent energy, J. Comput. Phys., 203 (2005), pp. 602-625.

[6] V. Caselles and F. Catte and T. Coll and F. Dibos, A geometric model for active contours in image processing, Numer. Math., 66 (1993), pp. 1-31.

[7] V. Caselles And B. Coll, Snakes in movement, SIAM J. Numer. Analy., 33 (1996), pp. 24452456.

[8] T. Chan and S. Esedoglu and M. Nikolova, Algorithms for Finding Global Minimizers of Image Segmentation and Denoising Models, SIAM J. Appl. Math., 66 (2006), pp. 16321648.

[9] V. Caselles and R. Kimmel and G. Sapiro, Geodesic Active Contours, International Journal of Computer Vision, 22 (1997), pp. 61-79.

[10] A. Chambolle, An algorithm for total variation minimization and applications, Journal of Mathematical Imaging and Vision, 19 (2004), pp. 89-97.

[11] T. Chan And B. Sandberg and L. Vese, Active contours without edges for vector-valued images, Journal of Visual Communication and Image Representation, 11 (2000), pp. 130141.

[12] T. Chan And L. Vese, An active contour model without edges, Scale-Space Theories in Computer Vision. Second International Conference, 1999.

[13] D.L. Chopp and J.A. Sethian, Motion by intrinsic Laplacian of curvature, Interfaces and Free Boundaries, 1 (1999), pp. 1-18.

[14] U. Clarenz, The Wulff-shape minimizes an anisotropic Willmore functional, Interfaces and Free Boundaries, 6 (2004), pp. 351-359.

[15] K. Deckelnick AND G. DziUK, Discrete anisotropic curvature flow of graphs, Mathematical Modelling and Numerical Analysis, 33 (1999), pp. 1203-1222.

[16] K. Deckelnick and G. Dziuk and C.M. Elliott, Fully discrete simi-implicit second order splitting for anisotropic surface diffusion of graphs, SIAM J. Numer. Anal., 43 (2005), pp. $1112-1138$.

[17] M. Droske And A. BerTozzI, Higher-order feature-preserving geometric regularization, SIAM J. Image Sci., 3 (2010), pp. 21-51.

[18] M. Elsey And S. Esedoglu, Analogue of the total variation denoising model in the context of geometry processing, UCLA CAM report 07-31, Los Angeles, CA, 2007.

[19] S. Esedoglu And S. Osher, Decomposition of images by the anisotropic Rudin-Osher-Fatemi model, Comm. Pure Appl. Math., 57 (2004), pp. 1609-1626.

[20] T. Goldstein And S. Osher, The split Bregman Method for $L_{1}$ regularized problems, UCLA CAM report 08-29, Los Angeles, CA, 2008.

[21] M. Kass and A. Witkin and D. Terzopoulos, Snakes: Active contour models, International Journal of Computer Vision, 1 (1998), pp. 321-331.

[22] S. Kichenassamy and A. Kumar and P. Olver and A. Tannenbaum and A. Yezzi, Gradient flows and geometric active contour models, Proceedings of the Fifth International Conference on Computer Vision, ICCV, 1995, pp. 810-815.

[23] D. Mumford and J. Shah, Optimal approximation by piecewise smooth functions and associated variational problems, Comm. Pure Appl. Math., 42 (1989), pp. 577-685.

[24] S. Osher And J. A. Sethian, Fronts propagating with curvature-dependent speed: Algorithms based on Hamilton-Jacobi Formulation, J. Comput. Phys., 79 (1988), pp. 12-49.

[25] P. Perona And J. Malik, Scale space and edge detection using anisotropic diffusion, IEEE Transactions on Pattern Analysis and Machine Intelligence, 12 (1990), pp. 629-639. 
[26] D. Salac And W. Lu, A local semi-implicit level-set method for interface motion, J. Sci. Comput., 35 (2008), pp. 330-349.

[27] G. SAPIRO, Vector snakes: a geometric framework for color, texture and multi-scale image segmentation, Proc. IEEE Int. Conf. Image Proc, 1996.

[28] C. Schoenlieb And A. Bertozzi, Unconditionally stable schemes for higher order inpainting, submitted to Comm. Math. Sci..

[29] P. Smereka, Semi-implicit level set methods for curvature and surface diffusion motion, J. Sci. Comput., 19 (2003), pp. 439-456.

[30] T.TAO, Nonlinear dispersive equations: local and global analysis, CBMS regional series in mathematics, 2006.

[31] J. Tumblin And G. Turk, A boundary hierarchy for detail-preserving contrast reduction, Proceedings of the SIGGRAPH annual conference on Computer Graphics, 1999, pp. 83-90.

[32] J. Ye, Applications of Variational Models in Geometric problems, Ph.D. Thesis, Department of Mathematics, UCLA, 2009. 